\title{
A Half-Discrete Hardy-Hilbert-Type Inequality with a Best Possible Constant Factor Related to the Hurwitz Zeta Function
}

Michael Th. Rassias and Bicheng Yang

\begin{abstract}
Using methods of weight functions, techniques of real analysis as well as the Hermite-Hadamard inequality, a half-discrete Hardy-Hilbert-type inequality with multi-parameters and a best possible constant factor related to the Hurwitz zeta function and the Riemann zeta function is obtained. Equivalent forms, normed operator expressions, their reverses and some particular cases are also considered.

Keywords Hardy-Hilbert-type inequality; Hurwitz zeta function; Riemann zeta function; weight function; operator

2000 Mathematics Subject Classification: 26D15 - 47A07 · 11Y35 - 31A10 . $65 \mathrm{~B} 10$
\end{abstract}

\section{Introduction}

$$
\begin{gathered}
\text { If } p>1, \frac{1}{p}+\frac{1}{q}=1, f(x), g(y) \geq 0, f \in L^{p}\left(\mathbf{R}_{+}\right), g \in L^{q}\left(\mathbf{R}_{+}\right), \\
\|f\|_{p}=\left(\int_{0}^{\infty} f^{p}(x) d x\right)^{\frac{1}{p}}>0,
\end{gathered}
$$

$\|g\|_{q}>0$, then we have the following Hardy-Hilbert's integral inequality (cf. [1]):

\footnotetext{
M. Th. Rassias

Department of Mathematics, ETH-Zürich, Rämistrasse 101, 8092 Zürich, Switzerland \& Department of Mathematics, Princeton University, Fine Hall, Washington Road, Princeton, NJ 08544-1000, USA

e-mail:michail.rassias@math.ethz.ch,michailrassias@math.princeton.edu

B. Yang

Department of Mathematics, Guangdong University of Education, Guangzhou,

Guangdong 510303, P. R. China,

e-mail: bcyang@gdei.edu.cn bcyang818@163.com
} 


$$
\int_{0}^{\infty} \int_{0}^{\infty} \frac{f(x) g(y)}{x+y} d x d y<\frac{\pi}{\sin (\pi / p)}\|f\|_{p}\|g\|_{q},
$$

where, the constant factor $\frac{\pi}{\sin (\pi / p)}$ is the best possible. Assuming that

$$
\begin{gathered}
a_{m}, b_{n} \geq 0, a=\left\{a_{m}\right\}_{m=1}^{\infty} \in l^{p}, b=\left\{b_{n}\right\}_{n=1}^{\infty} \in l^{q}, \\
\|a\|_{p}=\left(\sum_{m=1}^{\infty} a_{m}^{p}\right)^{\frac{1}{p}}>0,\|b\|_{q}>0,
\end{gathered}
$$

we have the following Hardy-Hilbert's inequality with the same best constant $\frac{\pi}{\sin (\pi / p)}$ (cf. [1]):

$$
\sum_{m=1}^{\infty} \sum_{n=1}^{\infty} \frac{a_{m} b_{n}}{m+n}<\frac{\pi}{\sin (\pi / p)}\|a\|_{p}\|b\|_{q} .
$$

Inequalities (1) and (2) are important in Analysis and its applications (cf. [1], [2], [3], [4], [5]).

$$
\begin{aligned}
& \text { If } \mu_{i}, v_{j}>0(i, j \in \mathbf{N}=\{1,2, \cdots\}), \\
& \qquad U_{m}:=\sum_{i=1}^{m} \mu_{i}, V_{n}:=\sum_{j=1}^{n} v_{j}(m, n \in \mathbf{N}),
\end{aligned}
$$

then we have the following inequality (cf. [1], Theorem 321) :

$$
\sum_{m=1}^{\infty} \sum_{n=1}^{\infty} \frac{\mu_{m}^{1 / q} v_{n}^{1 / p} a_{m} b_{n}}{U_{m}+V_{n}}<\frac{\pi}{\sin (\pi / p)}\|a\|_{p}\|b\|_{q}
$$

Replacing $\mu_{m}^{1 / q} a_{m}$ and $v_{n}^{1 / p} b_{n}$ by $a_{m}$ and $b_{n}$ in (4), respectively, we obtain the following equivalent form of (4):

$$
\sum_{m=1}^{\infty} \sum_{n=1}^{\infty} \frac{a_{m} b_{n}}{U_{m}+V_{n}}<\frac{\pi}{\sin \left(\frac{\pi}{p}\right)}\left(\sum_{m=1}^{\infty} \frac{a_{m}^{p}}{\mu_{m}^{p-1}}\right)^{\frac{1}{p}}\left(\sum_{n=1}^{\infty} \frac{b_{n}^{q}}{v_{n}^{q-1}}\right)^{\frac{1}{q}}
$$

For $\mu_{i}=v_{j}=1(i, j \in \mathbf{N})$, both (4) and (5) reduce to (2). We call (4) and (5) as Hardy-Hilbert-type inequalities.

Note. The authors did not prove that (4) is valid with the best possible constant factor in [1].

In 1998, by introducing an independent parameter $\lambda \in(0,1]$, Yang [6] gave an extension of (1) with the kernel $1 /(x+y)^{\lambda}$ for $p=q=2$. Optimizing the method used in [6], Yang [5] provided some extensions of (1) and (2) as follows:

If $\lambda_{1}, \lambda_{2} \in \mathbf{R}, \lambda_{1}+\lambda_{2}=\lambda, k_{\lambda}(x, y)$ is a non-negative homogeneous function of degree $-\lambda$, with

$$
\begin{array}{r}
k\left(\lambda_{1}\right)=\int_{0}^{\infty} k_{\lambda}(t, 1) t^{\lambda_{1}-1} d t \in \mathbf{R}_{+}, \\
\phi(x)=x^{p\left(1-\lambda_{1}\right)-1}, \psi(x)=x^{q\left(1-\lambda_{2}\right)-1}, f(x), g(y) \geq 0,
\end{array}
$$




$$
f \in L_{p, \phi}\left(\mathbf{R}_{+}\right)=\left\{f ;\|f\|_{p, \phi}:=\left\{\int_{0}^{\infty} \phi(x)|f(x)|^{p} d x\right\}^{\frac{1}{p}}<\infty\right\},
$$

$g \in L_{q, \psi}\left(\mathbf{R}_{+}\right),\|f\|_{p, \phi},\|g\|_{q, \psi}>0$, then we have

$$
\int_{0}^{\infty} \int_{0}^{\infty} k_{\lambda}(x, y) f(x) g(y) d x d y<k\left(\lambda_{1}\right)\|f\|_{p, \phi}\|g\|_{q, \psi},
$$

where, the constant factor $k\left(\lambda_{1}\right)$ is the best possible.

Moreover, if $k_{\lambda}(x, y)$ remains finite and $k_{\lambda}(x, y) x^{\lambda_{1}-1}\left(k_{\lambda}(x, y) y^{\lambda_{2}-1}\right)$ is decreasing with respect to $x>0(y>0)$, then for $a_{m}, b_{n} \geq 0$,

$$
a \in l_{p, \phi}=\left\{a ;\|a\|_{p, \phi}:=\left(\sum_{n=1}^{\infty} \phi(n)\left|a_{n}\right|^{p}\right)^{\frac{1}{p}}<\infty\right\},
$$

$b=\left\{b_{n}\right\}_{n=1}^{\infty} \in l_{q, \psi},\|a\|_{p, \phi},\|b\|_{q, \psi}>0$, we have

$$
\sum_{m=1}^{\infty} \sum_{n=1}^{\infty} k_{\lambda}(m, n) a_{m} b_{n}<k\left(\lambda_{1}\right)\|a\|_{p, \phi}\|b\|_{q, \psi}
$$

where, the constant factor $k\left(\lambda_{1}\right)$ is still the best possible.

Clearly, for

$$
\lambda=1, k_{1}(x, y)=\frac{1}{x+y}, \lambda_{1}=\frac{1}{q}, \lambda_{2}=\frac{1}{p},
$$

inequality (6) reduces to (1), while (7) reduces to (2). For

$$
0<\lambda_{1}, \lambda_{2} \leq 1, \lambda_{1}+\lambda_{2}=\lambda
$$

we set

$$
k_{\lambda}(x, y)=\frac{1}{(x+y)^{\lambda}}\left((x, y) \in \mathbf{R}_{+}^{2}\right) .
$$

Then by (7), we have

$$
\sum_{m=1}^{\infty} \sum_{n=1}^{\infty} \frac{a_{m} b_{n}}{(m+n)^{\lambda}}<B\left(\lambda_{1}, \lambda_{2}\right)\|a\|_{p, \phi}\|b\|_{q, \psi}
$$

where, the constant $B\left(\lambda_{1}, \lambda_{2}\right)$ is the best possible, and

$$
B(u, v)=\int_{0}^{\infty} \frac{1}{(1+t)^{u+v}} t^{u-1} d t(u, v>0)
$$

is the beta function.

In 2015, subject to further conditions, Yang [7] proved an extension of (8) and (5) as follows: 


$$
\begin{aligned}
& \sum_{m=1}^{\infty} \sum_{n=1}^{\infty} \frac{a_{m} b_{n}}{\left(U_{m}+V_{n}\right)^{\lambda}} \\
< & B\left(\lambda_{1}, \lambda_{2}\right)\left(\sum_{m=1}^{\infty} \frac{U_{m}^{p\left(1-\lambda_{1}\right)-1} a_{m}^{p}}{\mu_{m}^{p-1}}\right)^{\frac{1}{p}}\left(\sum_{n=1}^{\infty} \frac{V_{n}^{q\left(1-\lambda_{2}\right)-1} b_{n}^{q}}{v_{n}^{q-1}}\right)^{\frac{1}{q}},
\end{aligned}
$$

where, the constant $B\left(\lambda_{1}, \lambda_{2}\right)$ is still the best possible.

Further results including some multidimensional Hilbert-type inequalities can be found in [8]- [25].

On the topic of half-discrete Hilbert-type inequalities with non-homogeneous kernels, Hardy et al. provided a few results in Theorem 351 of [1]. But, they did not prove that the constant factors are the best possible. However, Yang [26] presented a result with the kernel $1 /(1+n x)^{\lambda}$ by introducing a variable and proved that the constant factor is the best possible. In 2011, Yang [27] gave the following halfdiscrete Hardy-Hilbert's inequality with the best possible constant factor $B\left(\lambda_{1}, \lambda_{2}\right)$ :

$$
\int_{0}^{\infty} f(x)\left[\sum_{n=1}^{\infty} \frac{a_{n}}{(x+n)^{\lambda}}\right] d x<B\left(\lambda_{1}, \lambda_{2}\right)\|f\|_{p, \phi}\|a\|_{q, \psi},
$$

where, $\lambda_{1}>0,0<\lambda_{2} \leq 1, \lambda_{1}+\lambda_{2}=\lambda$. Zhong et al. ([28]-[34]) investigated several half-discrete Hilbert-type inequalities with particular kernels.

Using methods of weight functions and techniques of discrete and integral Hilbert-type inequalities with some additional conditions on the kernel, a halfdiscrete Hilbert-type inequality with a general homogeneous kernel of degree $-\lambda \in$ $\mathbf{R}$ and a best constant factor $k\left(\lambda_{1}\right)$ is obtained as follows:

$$
\int_{0}^{\infty} f(x) \sum_{n=1}^{\infty} k_{\lambda}(x, n) a_{n} d x<k\left(\lambda_{1}\right)\|f\|_{p, \phi}\|a\|_{q, \psi},
$$

which is an extension of (11) ( cf. Yang and Chen [35]). Additionally, a half-discrete Hilbert-type inequality with a general non-homogeneous kernel and a best constant factor is given by Yang [36]. The reader is referred to the three books of Yang et al. [37], [38] and [39], where half-discrete Hilbert-type inequalities and their operator expressions are extensively treated.

In this paper, using methods of weight functions, techniques of real analysis as well as the Hermite-Hadamard inequality, a half-discrete Hardy-Hilbert-type inequality with multi-parameters and a best possible constant factor related to the Hurwitz zeta function and the Riemann zeta function is obtained, which is an extension of (12) for $\lambda=0$ in a particular kernel. Equivalent forms, normed operator expressions, their reverses and some particular cases are also considered. 


\section{An Example and Some Lemmas}

In the following, we assume that $\mu_{i}, v_{j}>0(i, j \in \mathbf{N}), U_{m}$ and $V_{n}$ are defined by (3),

$$
\widetilde{V}_{n}:=V_{n}-\widetilde{v}_{n}\left(\widetilde{v}_{n} \in\left[0, \frac{v_{n}}{2}\right]\right)(n \in \mathbf{N})
$$

$\mu(t)$ is a positive continuous function in $\mathbf{R}_{+}=(0, \infty)$,

$$
U(x):=\int_{0}^{x} \mu(t) d t<\infty(x \in[0, \infty))
$$

$v(t):=v_{n}, t \in\left(n-\frac{1}{2}, n+\frac{1}{2}\right](n \in \mathbf{N})$, and

$$
V(y):=\int_{\frac{1}{2}}^{y} v(t) d t\left(y \in\left[\frac{1}{2}, \infty\right)\right),
$$

$p \neq 0,1, \frac{1}{p}+\frac{1}{q}=1, \delta \in\{-1,1\}, f(x), a_{n} \geq 0\left(x \in \mathbf{R}_{+}, n \in \mathbf{N}\right)$

$$
\|f\|_{p, \Phi_{\delta}}=\left(\int_{0}^{\infty} \Phi_{\delta}(x) f^{p}(x) d x\right)^{\frac{1}{p}}
$$

$\|a\|_{q, \widetilde{\Psi}}=\left(\sum_{n=1}^{\infty} \widetilde{\Psi}(n) b_{n}^{q}\right)^{\frac{1}{q}}$, where,

$$
\Phi_{\delta}(x):=\frac{U^{p(1-\delta \sigma)-1}(x)}{\mu^{p-1}(x)}, \widetilde{\Psi}(n):=\frac{\widetilde{V}_{n}^{q(1-\sigma)-1}}{v_{n}^{q-1}}\left(x \in \mathbf{R}_{+}, n \in \mathbf{N}\right) .
$$

Example 1. For $0<\gamma<\sigma, 0 \leq \alpha \leq \rho(\rho>0)$,

$$
\operatorname{csch}(u):=\frac{2}{e^{u}-e^{-u}}(u>0)
$$

is the hyperbolic cosecant function (cf. [40]). We set

$$
h(t)=\frac{\operatorname{csch}\left(\rho t^{\gamma}\right)}{e^{\alpha t^{\gamma}}}\left(t \in \mathbf{R}_{+}\right) .
$$

(i) Setting $u=\rho t^{\gamma}$, we find 


$$
\begin{aligned}
k(\sigma) & :=\int_{0}^{\infty} \frac{\operatorname{csch}\left(\rho t^{\gamma}\right)}{e^{\alpha t^{\gamma}}} t^{\sigma-1} d t \\
& =\frac{1}{\gamma \rho^{\sigma / \gamma}} \int_{0}^{\infty} \frac{\csc h(u)}{e^{\frac{\alpha}{\rho} u}} u^{\frac{\sigma}{\gamma}-1} d u \\
& =\frac{2}{\gamma \rho^{\sigma / \gamma}} \int_{0}^{\infty} \frac{e^{-\frac{\alpha}{\rho} u} u^{\frac{\sigma}{\gamma}-1}}{e^{u}-e^{-u}} d u \\
& =\frac{2}{\gamma \rho^{\sigma / \gamma}} \int_{0}^{\infty} \frac{e^{-\left(\frac{\alpha}{\rho}+1\right) u} u^{\frac{\sigma}{\gamma}-1}}{1-e^{-2 u}} d u \\
& =\frac{2}{\gamma \rho^{\sigma / \gamma}} \int_{0}^{\infty} \sum_{k=0}^{\infty} e^{-\left(2 k+\frac{\alpha}{\rho}+1\right) u} u^{\frac{\sigma}{\gamma}-1} d u .
\end{aligned}
$$

By the Lebesgue term by term integration theorem (cf. [40]), setting $v=\left(2 k+\frac{\alpha}{\rho}+1\right) u$, we have

$$
\begin{aligned}
k(\sigma) & =\int_{0}^{\infty} \frac{\operatorname{csch}\left(\rho t^{\gamma}\right)}{e^{\alpha t \gamma}} t^{\sigma-1} d t \\
& =\frac{2}{\gamma \rho^{\sigma / \gamma}} \sum_{k=0}^{\infty} \int_{0}^{\infty} e^{-\left(2 k+\frac{\alpha}{\rho}+1\right) u} u^{\frac{\sigma}{\gamma}-1} d u \\
& =\frac{2}{\gamma \rho^{\sigma / \gamma}} \sum_{k=0}^{\infty} \frac{1}{\left(2 k+\frac{\alpha}{\rho}+1\right)^{\sigma / \gamma}} \int_{0}^{\infty} e^{-v} v^{\frac{\sigma}{\gamma}-1} d v \\
& =\frac{2 \Gamma\left(\frac{\sigma}{\gamma}\right)}{\gamma(2 \rho)^{\sigma / \gamma}} \sum_{k=0}^{\infty} \frac{1}{\left(k+\frac{\alpha+\rho}{2 \rho}\right)^{\sigma / \gamma}} \\
& =\frac{2 \Gamma\left(\frac{\sigma}{\gamma}\right)}{\gamma(2 \rho)^{\sigma / \gamma}} \zeta\left(\frac{\sigma}{\gamma}, \frac{\alpha+\rho}{2 \rho}\right) \in \mathbf{R}_{+},
\end{aligned}
$$

where,

$$
\zeta(s, a):=\sum_{k=0}^{\infty} \frac{1}{(k+a)^{s}}(s>1 ; 0<a \leq 1)
$$

is the Hurwitz zeta function, $\zeta(s)=\zeta(s, 1)$ is the Riemann zeta function, and

$$
\Gamma(y):=\int_{0}^{\infty} e^{-v} v^{y-1} d v(y>0)
$$

is the Gamma function (cf. [41]).

In particular, (1) for $\alpha=\rho>0$, we have $h(t)=\frac{\operatorname{csch}\left(\rho t^{\gamma}\right)}{e^{\rho t^{\gamma}}}$ and $k(\sigma)=\frac{2 \Gamma\left(\frac{\sigma}{\gamma}\right) \zeta\left(\frac{\sigma}{\gamma}\right)}{\gamma(2 \rho)^{\sigma / \gamma}}$. In this case, for $\gamma=\frac{\sigma}{2}$, we have $h(t)=\frac{\operatorname{csch}\left(\rho \sqrt{t^{\sigma}}\right)}{e^{\rho \sqrt{t^{\sigma}}}}$ and $k(\sigma)=\frac{\pi^{2}}{6 \sigma \rho^{2}} ;$ (2) for $\alpha=0$, we have $h(t)=\operatorname{csch}\left(\rho t^{\gamma}\right)$ and $\frac{2 \Gamma\left(\frac{\sigma}{\gamma}\right)}{\gamma(2 \rho)^{\sigma / \gamma}} \zeta\left(\frac{\sigma}{\gamma}, \frac{1}{2}\right)$. In this case, for $\gamma=\frac{\sigma}{2}$, we find $h(t)=\operatorname{csch}\left(\rho \sqrt{t^{\sigma}}\right)$ and $k(\sigma)=\frac{\pi^{2}}{2 \sigma \rho^{2}}$.

(ii) We obtain for $u>0, \frac{1}{e^{u}-e^{-u}}>0$, 


$$
\begin{aligned}
\frac{d}{d u}\left(\frac{1}{e^{u}-e^{-u}}\right) & =-\frac{e^{u}+e^{-u}}{\left(e^{u}-e^{-u}\right)^{2}}<0, \\
\frac{d^{2}}{d u^{2}}\left(\frac{1}{e^{u}-e^{-u}}\right) & =\frac{2\left(e^{u}+e^{-u}\right)^{2}-\left(e^{u}-e^{-u}\right)^{2}}{\left(e^{u}-e^{-u}\right)^{3}}>0 .
\end{aligned}
$$

If $g(u)>0, g^{\prime}(u)<0, g^{\prime \prime}(u)>0$, then for $0<\gamma \leq 1$,

$$
\begin{aligned}
g\left(\rho t^{\gamma}\right) & >0, \frac{d}{d t} g\left(\rho t^{\gamma}\right)=\rho \gamma t^{\gamma-1} g^{\prime}\left(\rho t^{\gamma}\right)<0, \\
\frac{d^{2}}{d t^{2}} g\left(\rho t^{\gamma}\right) & =\rho \gamma(\gamma-1) t^{\gamma-2} g^{\prime}\left(\rho t^{\gamma}\right)+\rho^{2} \gamma^{2} t^{2 \gamma-2} g^{\prime \prime}\left(\rho t^{\gamma}\right)>0 ;
\end{aligned}
$$

for $y \in\left(n-\frac{1}{2}, n+\frac{1}{2}\right), g(V(y))>0$,

$$
\begin{aligned}
\frac{d}{d y} g(V(y)) & =g^{\prime}(V(y)) v_{n}<0, \\
\frac{d^{2}}{d y^{2}} g(V(y)) & =g^{\prime \prime}(V(y)) v_{n}^{2}>0(n \in \mathbf{N}) .
\end{aligned}
$$

If $g_{i}(u)>0, g_{i}^{\prime}(u)<0, g_{i}^{\prime \prime}(u)>0(i=1,2)$, then

$$
\begin{aligned}
g_{1}(u) g_{2}(u) & >0, \\
\left(g_{1}(u) g_{2}(u)\right)^{\prime} & =g_{1}^{\prime}(u) g_{2}(u)+g_{1}(u) g_{2}^{\prime}(u)<0, \\
\left(g_{1}(u) g_{2}(u)\right)^{\prime \prime} & =g_{1}^{\prime \prime}(u) g_{2}(u)+2 g_{1}^{\prime}(u) g_{2}^{\prime}(u)+g_{1}(u) g_{2}^{\prime \prime}(u)>0(u>0) .
\end{aligned}
$$

(iii) Therefore, for $0<\gamma<\sigma \leq 1,0 \leq \alpha \leq \rho(\rho>0)$, we have $k(\sigma) \in \mathbf{R}_{+}$, with $h(t)>0, h^{\prime}(t)<0, h^{\prime \prime}(t)>0$, and then for $c>0, y \in\left(n-\frac{1}{2}, n+\frac{1}{2}\right)(n \in \mathbf{N})$, it follows that

$$
\begin{aligned}
& h(c V(y)) V^{\sigma-1}(y)>0, \\
& \frac{d}{d y} h(c V(y)) V^{\sigma-1}(y)<0, \\
& \frac{d^{2}}{d y^{2}} h(c V(y)) V^{\sigma-1}(y)>0 .
\end{aligned}
$$

Lemma 1. If $g(t)(>0)$ is decreasing in $\mathbf{R}_{+}$and strictly decreasing in $\left[n_{0}, \infty\right)$ where $n_{0} \in \mathbf{N}$, satisfying $\int_{0}^{\infty} g(t) d t \in \mathbf{R}_{+}$, then we have

$$
\int_{1}^{\infty} g(t) d t<\sum_{n=1}^{\infty} g(n)<\int_{0}^{\infty} g(t) d t .
$$

Proof. Since we have 


$$
\begin{aligned}
& \int_{n}^{n+1} g(t) d t \leq g(n) \leq \int_{n-1}^{n} g(t) d t\left(n=1, \cdots, n_{0}\right), \\
& \int_{n_{0}+1}^{n_{0}+2} g(t) d t<g\left(n_{0}+1\right)<\int_{n_{0}}^{n_{0}+1} g(t) d t,
\end{aligned}
$$

then it follows that

$$
0<\int_{1}^{n_{0}+2} g(t) d t<\sum_{n=1}^{n_{0}+1} g(n)<\sum_{n=1}^{n_{0}+1} \int_{n-1}^{n} g(t) d t=\int_{0}^{n_{0}+1} g(t) d t<\infty .
$$

Similarly, we still have

$$
0<\int_{n_{0}+2}^{\infty} g(t) d t \leq \sum_{n=n_{0}+2}^{\infty} g(n) \leq \int_{n_{0}+1}^{\infty} g(t) d t<\infty
$$

Hence, 14 follows and therefore the lemma is proved.

Lemma 2. If $0 \leq \alpha \leq \rho(\rho>0), 0<\gamma<\sigma \leq 1$, define the following weight coefficients:

$$
\begin{gathered}
\omega_{\delta}(\sigma, x):=\sum_{n=1}^{\infty} \frac{\operatorname{csch}\left(\rho\left(U^{\delta}(x) \widetilde{V}_{n}\right)^{\gamma}\right)}{e^{\alpha\left(U^{\delta}(x) \widetilde{V}_{n}\right)^{\gamma}}} \frac{U^{\delta \sigma}(x) v_{n}}{\widetilde{V}_{n}^{1-\sigma}}, x \in \mathbf{R}_{+}, \\
\varpi_{\delta}(\sigma, n):=\int_{0}^{\infty} \frac{\operatorname{csch}\left(\rho\left(U^{\delta}(x) \widetilde{V}_{n}\right)^{\gamma}\right)}{e^{\alpha\left(U^{\delta}(x) \widetilde{V}_{n}\right)^{\gamma}}} \frac{\widetilde{V}_{n}^{\sigma} \mu(x)}{U^{1-\delta \sigma}(x)} d x, n \in \mathbf{N} .
\end{gathered}
$$

Then, we have the following inequalities:

$$
\begin{aligned}
& \omega_{\delta}(\sigma, x)<k(\sigma)\left(x \in \mathbf{R}_{+}\right), \\
& \bar{\sigma}_{\delta}(\sigma, n) \leq k(\sigma)(n \in \mathbf{N}),
\end{aligned}
$$

where, $k(\sigma)$ is given by (13).

Proof. Since we find

$$
\begin{aligned}
\widetilde{V}_{n} & =V_{n}-\widetilde{v}_{n} \geq V_{n}-\frac{v_{n}}{2} \\
& =\int_{\frac{1}{2}}^{n+\frac{1}{2}} v(t) d t-\int_{n}^{n+\frac{1}{2}} v(t) d t=\int_{\frac{1}{2}}^{n} v(t) d t=V(n),
\end{aligned}
$$

and for $t \in\left(n-\frac{1}{2}, n+\frac{1}{2}\right], V^{\prime}(t)=v_{n}$, then by Example 1(iii) and Hermite-Hadamard's inequality (cf. [43]), we have 


$$
\begin{aligned}
& \frac{\operatorname{csch}\left(\rho\left(U^{\delta}(x) \widetilde{V}_{n}\right)^{\gamma}\right)}{e^{\alpha\left(U^{\delta}(x) \widetilde{V}_{n}\right)^{\gamma}}} \frac{v_{n}}{\widetilde{V}_{n}^{1-\sigma}} \\
\leq & \frac{\operatorname{csch}\left(\rho\left(U^{\delta}(x) V(n)\right)^{\gamma}\right)}{e^{\alpha\left(U^{\delta}(x) V(n)\right)^{\gamma}}} \frac{v_{n}}{V^{1-\sigma}(n)} \\
< & \int_{n-\frac{1}{2}}^{n+\frac{1}{2}} \frac{\operatorname{csch}\left(\rho\left(U^{\delta}(x) V(t)\right)^{\gamma}\right)}{e^{\alpha\left(U^{\delta}(x) V(t)\right)^{\gamma}}} \frac{V^{\prime}(t)}{V^{1-\sigma}(t)} d t, \\
\omega_{\delta}(\sigma, x)< & \sum_{n=1}^{\infty} \int_{n-\frac{1}{2}}^{n+\frac{1}{2}} \frac{\operatorname{csch}\left(\rho\left(U^{\delta}(x) V(t)\right)^{\gamma}\right)}{e^{\alpha\left(U^{\delta}(x) V(t)\right)^{\gamma}}} \frac{U^{\delta \sigma}(x) V^{\prime}(t)}{V^{1-\sigma}(t)} d t \\
= & \int_{\frac{1}{2}}^{\infty} \frac{\operatorname{csch}\left(\rho\left(U^{\delta}(x) V(t)\right)^{\gamma}\right)}{e^{\alpha\left(U^{\delta}(x) V(t)\right)^{\gamma}}} \frac{U^{\delta \sigma}(x) V^{\prime}(t)}{V^{1-\sigma}(t)} d t .
\end{aligned}
$$

Setting $u=U^{\delta}(x) V(t)$, by [13), we find

$$
\begin{aligned}
\omega_{\delta}(\sigma, x) & <\int_{0}^{U^{\delta}(x) V(\infty)} \frac{\operatorname{csch}\left(\rho u^{\gamma}\right)}{e^{\alpha u^{\gamma}}} \frac{U^{\delta \sigma}(x) U^{-\delta}(x)}{\left(u U^{-\delta}(x)\right)^{1-\sigma}} d u \\
& \leq \int_{0}^{\infty} \frac{\operatorname{csch}\left(\rho u^{\gamma}\right)}{e^{\alpha u^{\gamma}}} u^{\sigma-1} d u=k(\sigma) .
\end{aligned}
$$

Hence, 177 follows.

Setting $u=\widetilde{V}_{n} U^{\delta}(x)$ in 16 , we find $d u=\delta \widetilde{V}_{n} U^{\delta-1}(x) \mu(x) d x$ and

$$
\begin{aligned}
\varpi_{\delta}(\sigma, n) & =\frac{1}{\delta} \int_{\widetilde{V}_{n} U^{\delta}(0)}^{\widetilde{V}_{n} U^{\delta}(\infty)} \frac{\operatorname{csch}\left(\rho u^{\gamma}\right)}{e^{\alpha u^{\gamma}}} \frac{\widetilde{V}_{n}^{\sigma} \widetilde{V}_{n}^{-1}\left(\widetilde{V}_{n}^{-1} u\right)^{\frac{1}{\delta}-1}}{\left(\widetilde{V}_{n}^{-1} u\right)^{\frac{1}{\delta}-\sigma}} d u \\
& =\frac{1}{\delta} \int_{\widetilde{V}_{n} U^{\delta}(0)}^{\widetilde{V}_{n} U^{\delta}(\infty)} \frac{\operatorname{csch}\left(\rho u^{\gamma}\right)}{e^{\alpha u^{\gamma}}} u^{\sigma-1} d u .
\end{aligned}
$$

If $\delta=1$, then

$$
\begin{aligned}
\varpi_{1}(\sigma, n) & =\int_{0}^{\widetilde{V}_{n} U(\infty)} \frac{\operatorname{csch}\left(\rho u^{\gamma}\right)}{e^{\alpha u^{\gamma}}} u^{\sigma-1} d u \\
& \leq \int_{0}^{\infty} \frac{\operatorname{csch}\left(\rho u^{\gamma}\right)}{e^{\alpha u^{\gamma}}} u^{\sigma-1} d u .
\end{aligned}
$$

If $\delta=-1$, then

$$
\begin{aligned}
\varpi_{-1}(\sigma, n) & =-\int_{\infty}^{\widetilde{V}_{n} U^{-1}(\infty)} \frac{\operatorname{csch}\left(\rho u^{\gamma}\right)}{e^{\alpha u^{\gamma}}} u^{\sigma-1} d u \\
& \leq \int_{0}^{\infty} \frac{\operatorname{csch}\left(\rho u^{\gamma}\right)}{e^{\alpha u^{\gamma}}} u^{\sigma-1} d u .
\end{aligned}
$$

Then by (13), we have (18). The lemma is proved. 
Remark 1. We do not need the constraint $\sigma \leq 1$ to obtain (18). If $U(\infty)=\infty$, then we have

$$
\varpi_{\delta}(\sigma, n)=k(\sigma)(n \in \mathbf{N}) .
$$

For example, if we set $\mu(t)=\frac{1}{(1+t)^{\beta}}(t>0 ; 0 \leq \beta \leq 1)$, then for $x \geq 0$, we find

$$
\begin{aligned}
U(x) & =\int_{0}^{x} \frac{1}{(1+t)^{\beta}} d t \\
& =\left\{\begin{array}{c}
\frac{(1+x)^{1-\beta}-1}{1-\beta}, 0 \leq \beta<1<\infty, \\
\ln (1+x), \beta=1
\end{array}\right.
\end{aligned}
$$

and

$$
U(\infty)=\int_{0}^{\infty} \frac{1}{(1+t)^{\beta}} d t=\infty .
$$

Lemma 3. If $0 \leq \alpha \leq \rho(\rho>0), 0<\gamma<\sigma \leq 1$, there exists $n_{0} \in \mathbf{N}$, such that $v_{n} \geq v_{n+1}\left(n \in\left\{n_{0}, n_{0}+1, \cdots\right\}\right)$, and $V(\infty)=\infty$, then,

(i) for $x \in \mathbf{R}_{+}$, we have

$$
k(\sigma)\left(1-\theta_{\delta}(\sigma, x)\right)<\omega_{\delta}(\sigma, x),
$$

where, $\theta_{\delta}(\sigma, x)=O\left((U(x))^{\delta(\sigma-\gamma)}\right) \in(0,1)$;

(ii) for any $b>0$, we have

$$
\sum_{n=1}^{\infty} \frac{v_{n}}{\widetilde{V}_{n}^{1+b}}=\frac{1}{b}\left(\frac{1}{V_{n_{0}}^{b}}+b O(1)\right) .
$$

Proof. Since $v_{n} \geq v_{n+1}\left(n \geq n_{0}\right)$, and

$$
\widetilde{V}_{n}=V_{n}-\widetilde{v}_{n} \leq V_{n}=\int_{\frac{1}{2}}^{n+\frac{1}{2}} v(t) d t=V\left(n+\frac{1}{2}\right),
$$

by Example 1(iii), we have

$$
\begin{aligned}
\omega_{\delta}(\sigma, x) & =\sum_{n=1}^{\infty} \frac{\operatorname{csch}\left(\rho\left(U^{\delta}(x) \widetilde{V}_{n}\right)^{\gamma}\right)}{e^{\alpha\left(U^{\delta}(x) \widetilde{V}_{n}\right)^{\gamma}}} \frac{U^{\delta \sigma}(x) v_{n}}{\widetilde{V}_{n}^{1-\sigma}} \\
& \geq \sum_{n=n_{0}}^{\infty} \int_{n+\frac{1}{2}}^{n+\frac{3}{2}} \frac{\operatorname{csch}\left(\rho\left(U^{\delta}(x) V\left(n+\frac{1}{2}\right)\right)^{\gamma}\right)}{e^{\alpha\left(U^{\delta}(x) V\left(n+\frac{1}{2}\right)\right)^{\gamma}}} \frac{U^{\delta \sigma}(x) v_{n+1} d t}{\left(V\left(n+\frac{1}{2}\right)\right)^{1-\sigma}} \\
& >\sum_{n=n_{0}}^{\infty} \int_{n+\frac{1}{2}}^{n+\frac{3}{2}} \frac{\operatorname{csch}\left(\rho\left(U^{\delta}(x) V(t)\right)^{\gamma}\right)}{e^{\alpha\left(U^{\delta}(x) V(t)\right)^{\gamma}}} \frac{U^{\delta \sigma}(x) V^{\prime}(t)}{(V(t))^{1-\sigma}} d t \\
& =\int_{n_{0}+\frac{1}{2}}^{\infty} \frac{\operatorname{csch}\left(\rho\left(U^{\delta}(x) V(t)\right)^{\gamma}\right)}{e^{\alpha\left(U^{\delta}(x) V(t)\right)^{\gamma}}} \frac{U^{\delta \sigma}(x) V^{\prime}(t)}{(V(t))^{1-\sigma}} d t .
\end{aligned}
$$

Setting $u=U^{\delta}(x) V(t)$, in view of $V(\infty)=\infty$, by [13), we find 


$$
\begin{aligned}
\omega_{\delta}(\sigma, x) & >\int_{U^{\delta}(x) V_{n_{0}}}^{\infty} \frac{\operatorname{csch}\left(\rho u^{\gamma}\right)}{e^{\alpha u^{\gamma}}} u^{\sigma-1} d u \\
& =k(\sigma)-\int_{0}^{U^{\delta}(x) V_{n_{0}}} \frac{\operatorname{csch}\left(\rho u^{\gamma}\right)}{e^{\alpha u^{\gamma}}} u^{\sigma-1} d u \\
& =k(\sigma)\left(1-\theta_{\delta}(\sigma, x)\right), \\
\theta_{\delta}(\sigma, x) & :=\frac{1}{k(\sigma)} \int_{0}^{U^{\delta}(x) V_{n_{0}}} \frac{\operatorname{csch}\left(\rho u^{\gamma}\right)}{e^{\alpha u^{\gamma}}} u^{\sigma-1} d u \in(0,1) .
\end{aligned}
$$

Since $F(u)=\frac{u^{\gamma} \operatorname{csch}\left(\rho u^{\gamma}\right)}{e^{\alpha u^{\gamma}}}$ is continuous in $(0, \infty)$, satisfying

$$
F(u) \rightarrow \frac{1}{\rho}\left(u \rightarrow 0^{+}\right), F(u) \rightarrow 0(u \rightarrow \infty),
$$

there exists a constant $L>0$, such that $F(u) \leq L$, namely,

$$
\frac{\operatorname{csch}\left(\rho u^{\gamma}\right)}{e^{\alpha u^{\gamma}}} \leq L u^{-\gamma}(u \in(0, \infty))
$$

Hence we find

$$
\begin{aligned}
0 & <\theta_{\delta}(\sigma, x) \leq \frac{L}{k(\sigma)} \int_{0}^{U^{\delta}(x) V_{n_{0}}} u^{\sigma-\gamma-1} d u \\
& =\frac{L\left(U^{\delta}(x) V_{n_{0}}\right)^{\sigma-\gamma}}{k(\sigma)(\sigma-\gamma)}
\end{aligned}
$$

and then (20) follows.

For $b>0$, we find

$$
\begin{aligned}
\sum_{n=1}^{\infty} \frac{v_{n}}{\widetilde{V}_{n}^{1+b}} & \leq \sum_{n=1}^{n_{0}} \frac{v_{n}}{\widetilde{V}_{n}^{1+b}}+\sum_{n=n_{0}+1}^{\infty} \frac{v_{n}}{V^{1+b}(n)} \\
& <\sum_{n=1}^{n_{0}} \frac{v_{n}}{\widetilde{V}_{n}^{1+b}}+\sum_{n=n_{0}+1}^{\infty} \int_{n-\frac{1}{2}}^{n+\frac{1}{2}} \frac{V^{\prime}(x)}{V^{1+b}(x)} d x \\
& =\sum_{n=1}^{n_{0}} \frac{v_{n}}{\widetilde{V}_{n}^{1+b}}+\int_{n_{0}+\frac{1}{2}}^{\infty} \frac{d V(x)}{V^{1+b}(x)} \\
& =\sum_{n=1}^{n_{0}} \frac{v_{n}}{\widetilde{V}_{n}^{1+b}}+\frac{1}{b V^{b}\left(n_{0}+\frac{1}{2}\right)} \\
& =\frac{1}{b}\left(\frac{1}{V_{n_{0}}^{b}}+b \sum_{n=1}^{n_{0}} \frac{v_{n}}{\widetilde{V}_{n}^{1+b}}\right),
\end{aligned}
$$




$$
\begin{aligned}
\sum_{n=1}^{\infty} \frac{v_{n}}{\widetilde{V}_{n}^{1+b}} & \geq \sum_{n=n_{0}}^{\infty} \int_{n+\frac{1}{2}}^{n+\frac{3}{2}} \frac{v_{n+1}}{V^{1+b}\left(n+\frac{1}{2}\right)} d x \\
& >\sum_{n=n_{0}}^{\infty} \int_{n+\frac{1}{2}}^{n+\frac{3}{2}} \frac{V^{\prime}(x)}{V^{1+b}(x)} d x=\int_{n_{0}+\frac{1}{2}}^{\infty} \frac{d V(x)}{V^{1+b}(x)} \\
& =\frac{1}{b V^{b}\left(n_{0}+\frac{1}{2}\right)}=\frac{1}{b V_{n_{0}}^{b}} .
\end{aligned}
$$

Hence we have (21). The lemma is proved.

Note. For example, $v_{n}=\frac{1}{(n-\tau)^{\beta}}(n \in \mathbf{N} ; 0 \leq \beta \leq 1,0 \leq \tau<1)$ satisfies the conditions of Lemma 3 (for $n_{0} \geq 1$ ).

\section{Equivalent Inequalities and Operator Expressions}

Theorem 1. If $0 \leq \alpha \leq \rho(\rho>0), 0<\gamma<\sigma \leq 1, k(\sigma)$ is given by (13), then for $p>1,0<\|f\|_{p, \Phi_{\delta}},\|a\|_{q, \widetilde{\Psi}}<\infty$, we have the following equivalent inequalities:

$$
\begin{aligned}
I & :=\sum_{n=1}^{\infty} \int_{0}^{\infty} \frac{\operatorname{csch}\left(\rho\left(U^{\delta}(x) \widetilde{V}_{n}\right)^{\gamma}\right)}{e^{\alpha\left(U^{\delta}(x) \widetilde{V}_{n}\right)^{\gamma}}} a_{n} f(x) d x<k(\sigma)\|f\|_{p, \Phi_{\delta}}\|a\|_{q, \widetilde{\Psi}} \\
J_{1} & :=\sum_{n=1}^{\infty} \frac{v_{n}}{\widetilde{V}_{n}^{1-p \sigma}}\left[\int_{0}^{\infty} \frac{\operatorname{csch}\left(\rho\left(U^{\delta}(x) \widetilde{V}_{n}\right)^{\gamma}\right)}{e^{\alpha\left(U^{\delta}(x) \widetilde{V}_{n}\right)^{\gamma}}} f(x) d x\right]^{p} \\
& <k(\sigma)\|f\|_{p, \Phi_{\delta}} \\
J_{2} & :=\left\{\int_{0}^{\infty} \frac{\mu(x)}{U^{1-q \delta \sigma}(x)}\left[\sum_{n=1}^{\infty} \frac{\operatorname{csch}\left(\rho\left(U^{\delta}(x) \widetilde{V}_{n}\right)^{\gamma}\right)}{e^{\alpha\left(U^{\delta}(x) \widetilde{V}_{n}\right)^{\gamma}}} a_{n}\right]^{q} d x\right\}^{\frac{1}{q}} \\
& <k(\sigma)\|a\|_{q, \widetilde{\Psi}} .
\end{aligned}
$$

Proof. By Hölder's inequality with weight (cf. [43]), we have 


$$
\begin{aligned}
& {\left[\int_{0}^{\infty} \frac{\operatorname{csch}\left(\rho\left(U^{\delta}(x) \widetilde{V}_{n}\right)^{\gamma}\right)}{e^{\alpha\left(U^{\delta}(x) \widetilde{V}_{n}\right)^{\gamma}}} f(x) d x\right]^{p}} \\
& =\left[\int_{0}^{\infty} \frac{\operatorname{csch}\left(\rho\left(U^{\delta}(x) \widetilde{V}_{n}\right)^{\gamma}\right)}{e^{\alpha\left(U^{\delta}(x) \widetilde{V}_{n}\right)^{\gamma}}}\left(\frac{U^{\frac{1-\delta \sigma}{q}}(x) f(x)}{\widetilde{V}_{n}^{\frac{1-\sigma}{p}} \mu^{\frac{1}{q}}(x)}\right)\left(\frac{\widetilde{V}_{n}^{\frac{1-\sigma}{p}} \mu^{\frac{1}{q}}(x)}{U^{\frac{1-\delta \sigma}{q}}(x)}\right) d x\right]^{p} \\
& \leq \int_{0}^{\infty} \frac{\operatorname{csch}\left(\rho\left(U^{\delta}(x) \widetilde{V}_{n}\right)^{\gamma}\right)}{e^{\alpha\left(U^{\delta}(x) \widetilde{V}_{n}\right)^{\gamma}}}\left(\frac{U^{\frac{p(1-\delta \sigma)}{q}}(x) f^{p}(x)}{\widetilde{V}_{n}^{1-\sigma} \mu^{\frac{p}{q}}(x)}\right) d x \\
& \times\left[\int_{0}^{\infty} \frac{\operatorname{csch}\left(\rho\left(U^{\delta}(x) \widetilde{V}_{n}\right)^{\gamma}\right)}{e^{\alpha\left(U^{\delta}(x) \widetilde{V}_{n}\right)^{\gamma}}} \frac{\widetilde{V}_{n}^{(1-\sigma)(p-1)} \mu(x)}{U^{1-\delta \sigma}(x)} d x\right]^{p-1} \\
& =\frac{\left(\varpi_{\delta}(\sigma, n)\right)^{p-1}}{\widetilde{V}_{n}^{p \sigma-1} v_{n}} \int_{0}^{\infty} \frac{\operatorname{csch}\left(\rho\left(U^{\delta}(x) \widetilde{V}_{n}\right)^{\gamma}\right)}{e^{\alpha\left(U^{\delta}(x) \widetilde{V}_{n}\right)^{\gamma}}} \frac{U^{(1-\delta \sigma)(p-1)}(x) v_{n}}{\widetilde{V}_{n}^{1-\sigma} \mu^{p-1}(x)} f^{p}(x) d x .
\end{aligned}
$$

In view of (18) and the Lebesgue term by term integration theorem (cf. [42]), we find

$$
\begin{aligned}
J_{1} & \leq(k(\sigma))^{\frac{1}{q}}\left[\sum_{n=1}^{\infty} \int_{0}^{\infty} \frac{\operatorname{csch}\left(\rho\left(U^{\delta}(x) \widetilde{V}_{n}\right)^{\gamma}\right)}{e^{\alpha\left(U^{\delta}(x) \widetilde{V}_{n}\right)^{\gamma}}} \frac{U^{(1-\delta \sigma)(p-1)}(x) v_{n}}{\widetilde{V}_{n}^{1-\sigma} \mu^{p-1}(x)} f^{p}(x) d x\right]^{\frac{1}{p}} \\
& =(k(\sigma))^{\frac{1}{q}}\left[\int_{0}^{\infty} \sum_{n=1}^{\infty} \frac{\operatorname{csch}\left(\rho\left(U^{\delta}(x) \widetilde{V}_{n}\right)^{\gamma}\right)}{e^{\alpha\left(U^{\delta}(x) \widetilde{V}_{n}\right)^{\gamma}}} \frac{U^{(1-\delta \sigma)(p-1)}(x) v_{n}}{\widetilde{V}_{n}^{1-\sigma} \mu^{p-1}(x)} f^{p}(x) d x\right]^{\frac{1}{p}} \\
& =(k(\sigma))^{\frac{1}{q}}\left[\int_{0}^{\infty} \omega_{\delta}(\sigma, x) \frac{U^{p(1-\delta \sigma)-1}(x)}{\mu^{p-1}(x)} f^{p}(x) d x\right]^{\frac{1}{p}} .
\end{aligned}
$$

Then by 177, we have (23).

By Hölder's inequality (cf. [43]), we have

$$
\begin{aligned}
I & =\sum_{n=1}^{\infty}\left[\frac{v_{n}^{\frac{1}{p}}}{\widetilde{V}_{n}^{\frac{1}{p}-\sigma}} \int_{0}^{\infty} \frac{\operatorname{csch}\left(\rho\left(U^{\delta}(x) \widetilde{V}_{n}\right)^{\gamma}\right)}{e^{\alpha\left(U^{\delta}(x) \widetilde{V}_{n}\right)^{\gamma}}} f(x) d x\right]\left(\frac{\widetilde{V}_{n}^{\frac{1}{p}-\sigma} a_{n}}{v_{n}^{\frac{1}{p}}}\right) \\
& \leq J_{1}\|a\|_{q, \widetilde{\Psi}} .
\end{aligned}
$$

Then by (23), we have (22).

On the other hand, assuming that (22) is valid, we set

$$
a_{n}:=\frac{v_{n}}{\widetilde{V}_{n}^{1-p \sigma}}\left[\int_{0}^{\infty} \frac{\operatorname{csch}\left(\rho\left(U^{\delta}(x) \widetilde{V}_{n}\right)^{\gamma}\right)}{e^{\alpha\left(U^{\delta}(x) \widetilde{V}_{n}\right)^{\gamma}}} f(x) d x\right]^{p-1}, n \in \mathbf{N} .
$$

Then, we find $J_{1}^{p}=\|a\|_{q, \widetilde{\Psi}}^{q}$.

If $J_{1}=0$, then (23) is trivially valid. 
If $J_{1}=\infty$, then (23) keeps impossible.

Suppose that $0<J_{1}<\infty$. By [22], it follows that

$$
\begin{aligned}
& \|a\|_{q, \widetilde{\Psi}}^{q}=J_{1}^{p}=I<k(\sigma)\|f\|_{p, \Phi_{\delta}}\|a\|_{q, \widetilde{\Psi}}, \\
& \|a\|_{q, \widetilde{\Psi}}^{q-1}=J_{1}<k(\sigma)\|f\|_{p, \Phi_{\delta}},
\end{aligned}
$$

and then (23) follows, which is equivalent to (22).

By Hölder's inequality with weight (cf. [43]), we obtain

$$
\begin{aligned}
& {\left[\sum_{n=1}^{\infty} \frac{\operatorname{csch}\left(\rho\left(U^{\delta}(x) \widetilde{V}_{n}\right)^{\gamma}\right)}{e^{\alpha\left(U^{\delta}(x) \widetilde{V}_{n}\right)^{\gamma}}} a_{n}\right]^{q}} \\
& =\left[\sum_{n=1}^{\infty} \frac{\operatorname{csch}\left(\rho\left(U^{\delta}(x) \widetilde{V}_{n}\right)^{\gamma}\right)}{e^{\alpha\left(U^{\delta}(x) \widetilde{V}_{n}\right)^{\gamma}}}\left(\frac{U^{\frac{1-\delta \sigma}{q}}(x) v_{n}^{\frac{1}{p}}}{\widetilde{V}_{n}^{\frac{1-\sigma}{p}}}\right)\left(\frac{\widetilde{V}_{n}^{\frac{1-\sigma}{p}} a_{n}}{U^{\frac{1-\delta \sigma}{q}}(x) v_{n}^{\frac{1}{p}}}\right)\right]^{q} \\
& \leq\left[\sum_{n=1}^{\infty} \frac{\operatorname{csch}\left(\rho\left(U^{\delta}(x) \widetilde{V}_{n}\right)^{\gamma}\right)}{e^{\alpha\left(U^{\delta}(x) \widetilde{V}_{n}\right)^{\gamma}}} \frac{U^{(1-\delta \sigma)(p-1)}(x) v_{n}}{\widetilde{V}_{n}^{1-\sigma}}\right]^{q-1} \\
& \times \sum_{n=1}^{\infty} \frac{\operatorname{csch}\left(\rho\left(U^{\delta}(x) \widetilde{V}_{n}\right)^{\gamma}\right)}{e^{\alpha\left(U^{\delta}(x) \widetilde{V}_{n}\right)^{\gamma}}} \frac{\widetilde{V}_{n}^{\frac{q(1-\sigma)}{p}}}{U^{1-\delta \sigma}(x) v_{n}^{q-1}} a_{n}^{q} \\
& =\frac{\left(\omega_{\delta}(\sigma, x)\right)^{q-1}}{U^{q \delta \sigma-1}(x) \mu(x)} \sum_{n=1}^{\infty} \frac{\operatorname{csch}\left(\rho\left(U^{\delta}(x) \widetilde{V}_{n}\right)^{\gamma}\right)}{e^{\alpha\left(U^{\delta}(x) \widetilde{V}_{n}\right)^{\gamma}}} \frac{\widetilde{V}_{n}^{(1-\sigma)(q-1)} \mu(x)}{U^{1-\delta \sigma}(x) v_{n}^{q-1}} a_{n}^{q} .
\end{aligned}
$$

Then by (17) and Lebesgue term by term integration theorem (cf. [42]), it follows that

$$
\begin{aligned}
J_{2} & <(k(\sigma))^{\frac{1}{p}}\left\{\int_{0}^{\infty} \sum_{n=1}^{\infty} \frac{\operatorname{csch}\left(\rho\left(U^{\delta}(x) \widetilde{V}_{n}\right)^{\gamma}\right)}{e^{\alpha\left(U^{\delta}(x) \widetilde{V}_{n}\right)^{\gamma}}} \frac{\widetilde{V}_{n}^{(1-\sigma)(q-1)} \mu(x)}{U^{1-\delta \sigma}(x) v_{n}^{q-1}} a_{n}^{q} d x\right\}^{\frac{1}{q}} \\
& =(k(\sigma))^{\frac{1}{p}}\left\{\sum_{n=1}^{\infty} \int_{0}^{\infty} \frac{\operatorname{csch}\left(\rho\left(U^{\delta}(x) \widetilde{V}_{n}\right)^{\gamma}\right)}{e^{\alpha\left(U^{\delta}(x) \widetilde{V}_{n}\right)^{\gamma}}} \frac{\widetilde{V}_{n}^{(1-\sigma)(q-1)} \mu(x)}{U^{1-\delta \sigma}(x) v_{n}^{q-1}} a_{n}^{q} d x\right\}^{\frac{1}{q}} \\
& =(k(\sigma))^{\frac{1}{p}}\left\{\sum_{n=1}^{\infty} \varpi_{\delta}(\sigma, n) \frac{\widetilde{V}_{n}^{q(1-\sigma)-1}}{v_{n}^{q-1}} a_{n}^{q}\right\}^{\frac{1}{q}} .
\end{aligned}
$$

Then by (18), we have (24).

By Hölder's inequality (cf. [43]), we have

$$
\begin{aligned}
I & =\int_{0}^{\infty}\left(\frac{U^{\frac{1}{q}-\delta \sigma}(x)}{\mu^{\frac{1}{q}}(x)} f(x)\right)\left[\frac{\mu^{\frac{1}{q}}(x)}{U^{\frac{1}{q}-\delta \sigma}(x)} \sum_{n=1}^{\infty} \frac{\operatorname{csch}\left(\rho\left(U^{\delta}(x) \widetilde{V}_{n}\right)^{\gamma}\right)}{e^{\alpha\left(U^{\delta}(x) \widetilde{V}_{n}\right)^{\gamma}}} a_{n}\right] d x \\
& \leq\|f\|_{p, \Phi_{\delta}} J_{2} .
\end{aligned}
$$


Then by 24), we have (22).

On the other hand, assuming that (24) is valid, we set

$$
f(x):=\frac{\mu(x)}{U^{1-q \delta \sigma}(x)}\left[\sum_{n=1}^{\infty} \frac{\operatorname{csch}\left(\rho\left(U^{\delta}(x) \widetilde{V}_{n}\right)^{\gamma}\right)}{e^{\alpha\left(U^{\delta}(x) \widetilde{V}_{n}\right)^{\gamma}}} a_{n}\right]^{q-1}, x \in \mathbf{R}_{+} .
$$

Then we find $J_{2}^{q}=\|f\|_{p, \Phi_{\delta}}^{p}$.

If $J_{2}=0$, then (24) is trivially valid.

If $J_{2}=\infty$, then (24) keeps impossible.

Suppose that $0<J_{2}<\infty$. By [22), it follows that

$$
\begin{aligned}
& \|f\|_{p, \Phi_{\delta}}^{p}=J_{2}^{q}=I<k(\sigma)\|f\|_{p, \Phi_{\delta}}\|a\|_{q, \widetilde{\Psi}}, \\
& \|f\|_{p, \Phi_{\delta}}^{p-1}=J_{2}<k(\sigma)\|a\|_{q, \widetilde{\Psi}},
\end{aligned}
$$

and then (24) follows, which is equivalent to (22).

Therefore, (22), (23) and (24) are equivalent. The theorem is proved.

Theorem 2. With the assumptions of Theorem 1, if there exists $n_{0} \in \mathbf{N}$, such that $v_{n} \geq v_{n+1}\left(n \in\left\{n_{0}, n_{0}+1, \cdots\right\}\right)$, and $U(\infty)=V(\infty)=\infty$, then the constant factor $k(\sigma)$ in (22), (23) and (24) is the best possible.

Proof. For $\varepsilon \in(0, q(\sigma-\gamma))$, we set $\widetilde{\sigma}=\sigma-\frac{\varepsilon}{q}(\in(\gamma, 1))$, and $\widetilde{f}=\widetilde{f}(x), x \in \mathbf{R}_{+}, \widetilde{a}=$ $\left\{\widetilde{a}_{n}\right\}_{n=1}^{\infty}$,

$$
\begin{aligned}
\widetilde{f}(x) & =\left\{\begin{array}{c}
U^{\delta(\widetilde{\sigma}+\varepsilon)-1}(x) \mu(x), 0<x^{\delta} \leq 1 \\
0, x^{\delta}>0
\end{array}\right. \\
\widetilde{a}_{n} & =\widetilde{V}_{n}^{\widetilde{\sigma}-1} v_{n}=\widetilde{V}_{n}^{\sigma-\frac{\varepsilon}{q}-1} v_{n}, n \in \mathbf{N} .
\end{aligned}
$$

Then for $\delta= \pm 1$, since $U(\infty)=\infty$, we find

$$
\int_{\left\{x>0 ; 0<x^{\delta} \leq 1\right\}} \frac{\mu(x)}{U^{1-\delta \varepsilon}(x)} d x=\frac{1}{\varepsilon} U^{\delta \varepsilon}(1) .
$$

By (21), (33) and (20), we obtain

$$
\begin{aligned}
\|\widetilde{f}\|_{p, \Phi_{\delta}}\|\widetilde{a}\|_{q, \widetilde{\Psi}} & =\left(\int_{\left\{x>0 ; 0<x^{\delta} \leq 1\right\}} \frac{\mu(x) d x}{U^{1-\delta \varepsilon}(x)}\right)^{\frac{1}{p}}\left(\sum_{n=1}^{\infty} \frac{v_{n}}{\widetilde{V}_{n}^{1+\varepsilon}}\right)^{\frac{1}{q}} \\
& =\frac{1}{\varepsilon} U^{\frac{\delta \varepsilon}{p}}(1)\left(\frac{1}{V_{n_{0}}^{\varepsilon}}+\varepsilon \widetilde{O}(1)\right)^{\frac{1}{q}},
\end{aligned}
$$




$$
\begin{aligned}
& \widetilde{I}:=\int_{0}^{\infty} \sum_{n=1}^{\infty} \frac{\operatorname{csch}\left(\rho\left(U^{\delta}(x) \widetilde{V}_{n}\right)^{\gamma}\right)}{e^{\alpha\left(U^{\delta}(x) \widetilde{V}_{n}\right)^{\gamma}}} \widetilde{a}_{n} \widetilde{f}(x) d x \\
& =\int_{\left\{x>0 ; 0<x^{\delta} \leq 1\right\}} \sum_{n=1}^{\infty} \frac{\operatorname{csch}\left(\rho\left(U^{\delta}(x) \widetilde{V}_{n}\right)^{\gamma}\right)}{e^{\alpha\left(U^{\delta}(x) \widetilde{V}_{n}\right)^{\gamma}}} \frac{\widetilde{V}_{n}^{\widetilde{\sigma}-1} v_{n} \mu(x)}{U^{1-\delta(\widetilde{\sigma}+\varepsilon)}(x)} d x \\
& =\int_{\left\{x>0 ; 0<x^{\delta} \leq 1\right\}} \omega_{\delta}(\widetilde{\sigma}, x) \frac{\mu(x)}{U^{1-\delta \varepsilon}(x)} d x \\
& \geq k(\widetilde{\sigma}) \int_{\left\{x>0 ; 0<x^{\delta} \leq 1\right\}}\left(1-\theta_{\delta}(\widetilde{\sigma}, x)\right) \frac{\mu(x)}{U^{1-\delta \varepsilon}(x)} d x \\
& =k(\widetilde{\sigma}) \int_{\left\{x>0 ; 0<x^{\delta} \leq 1\right\}}\left(1-O\left((U(x))^{\delta\left(\sigma-\frac{\varepsilon}{q}-\gamma\right)}\right)\right) \frac{\mu(x)}{U^{1-\delta \varepsilon}(x)} d x \\
& =k(\widetilde{\sigma})\left[\int_{\left\{x>0 ; 0<x^{\delta} \leq 1\right\}} \frac{\mu(x)}{U^{1-\delta \varepsilon}(x)} d x\right. \\
& \left.-\int_{\left\{x>0 ; 0<x^{\delta} \leq 1\right\}} O\left(\frac{\mu(x)}{U^{1-\delta\left(\sigma-\gamma+\frac{\varepsilon}{p}\right)}(x)}\right) d x\right] \\
& =\frac{1}{\varepsilon} k\left(\sigma-\frac{\varepsilon}{q}\right)\left(U^{\delta \varepsilon}(1)-\varepsilon O(1)\right) .
\end{aligned}
$$

If there exists a positive constant $K \leq k(\sigma)$, such that 22) is valid when replacing $k(\sigma)$ to $K$, then in particular, by Lebesgue term by term integration theorem, we have $\varepsilon \widetilde{I}<\varepsilon K\|\widetilde{f}\|_{p, \Phi_{\delta}}|| \widetilde{a} \|_{q, \widetilde{\Psi}}$, namely,

$$
k\left(\sigma-\frac{\varepsilon}{q}\right)\left(U^{\delta \varepsilon}(1)-\varepsilon O(1)\right)<K \cdot U^{\frac{\delta \varepsilon}{p}}(1)\left(\frac{1}{V_{n_{0}}^{\varepsilon}}+\varepsilon \widetilde{O}(1)\right)^{\frac{1}{q}}
$$

It follows that $k(\sigma) \leq K\left(\varepsilon \rightarrow 0^{+}\right)$. Hence, $K=k(\sigma)$ is the best possible constant factor of 22.

The constant factor $k(\sigma)$ in (23) (24) is still the best possible. Otherwise, we would reach a contradiction by (27) (20) that the constant factor in (22) is not the best possible. The theorem is proved.

For $p>1$, we find

$$
\widetilde{\Psi}^{1-p}(n)=\frac{v_{n}}{\widetilde{V}_{n}^{1-p \sigma}}(n \in \mathbf{N}), \Phi_{\delta}^{1-q}(x)=\frac{\mu(x)}{U^{1-q \delta \sigma}(x)}\left(x \in \mathbf{R}_{+}\right),
$$

and define the following real normed spaces:

$$
\begin{aligned}
L_{p, \Phi_{\delta}}\left(\mathbf{R}_{+}\right) & =\left\{f ; f=f(x), x \in \mathbf{R}_{+},\|f\|_{p, \Phi_{\delta}}<\infty\right\}, \\
l_{q, \widetilde{\Psi}} & =\left\{a ; a=\left\{a_{n}\right\}_{n=1}^{\infty},\|a\|_{q, \widetilde{\Psi}}<\infty\right\}, \\
L_{q, \Phi_{\delta}^{1-q}}\left(\mathbf{R}_{+}\right) & =\left\{h ; h=h(x), x \in \mathbf{R}_{+},\|h\|_{q, \Phi_{\delta}^{1-q}}<\infty\right\}, \\
l_{p, \widetilde{\Psi}^{1-p}} & =\left\{c ; c=\left\{c_{n}\right\}_{n=1}^{\infty},\|c\|_{p, \widetilde{\Psi}^{1-p}}<\infty\right\} .
\end{aligned}
$$


Assuming that $f \in L_{p, \Phi_{\delta}}\left(\mathbf{R}_{+}\right)$, setting

$$
c=\left\{c_{n}\right\}_{n=1}^{\infty}, c_{n}:=\int_{0}^{\infty} \frac{\operatorname{csch}\left(\rho\left(U^{\delta}(x) \widetilde{V}_{n}\right)^{\gamma}\right)}{e^{\alpha\left(U^{\delta}(x) \widetilde{V}_{n}\right)^{\gamma}}} f(x) d x, n \in \mathbf{N},
$$

we can rewrite 23 as follows:

$$
\left\|c||_{p, \widetilde{\Psi}^{1-p}}<k(\sigma)|| f\right\|_{p, \Phi_{\delta}}<\infty
$$

namely, $c \in l_{p, \widetilde{\Psi}^{1-p}}$.

Definition 1. Define a half-discrete Hardy-Hilbert-type operator

$$
T_{1}: L_{p, \Phi_{\delta}}\left(\mathbf{R}_{+}\right) \rightarrow l_{p, \widetilde{\Psi}^{1-p}}
$$

as follows:

For any $f \in L_{p, \Phi_{\delta}}\left(\mathbf{R}_{+}\right)$, there exists a unique representation $T_{1} f=c \in l_{p, \widetilde{\Psi}^{1-p}}$. Define the formal inner product of $T_{1} f$ and $a=\left\{a_{n}\right\}_{n=1}^{\infty} \in l_{q, \widetilde{\Psi}}$ as follows:

$$
\left(T_{1} f, a\right):=\sum_{n=1}^{\infty}\left[\int_{0}^{\infty} \frac{\operatorname{csch}\left(\rho\left(U^{\delta}(x) \widetilde{V}_{n}\right)^{\gamma}\right)}{e^{\alpha\left(U^{\delta}(x) \widetilde{V}_{n}\right)^{\gamma}}} f(x) d x\right] a_{n} .
$$

Then we can rewrite (22) and (23) as:

$$
\begin{aligned}
\left(T_{1} f, a\right) & <k(\sigma)\|f\|_{p, \Phi_{\delta}}\|a\|_{q, \widetilde{\Psi}}, \\
\left\|T_{1} f\right\|_{p, \widetilde{\Psi}^{1-p}} & <k(\sigma)\|f\|_{p, \Phi_{\delta}} .
\end{aligned}
$$

Define the norm of operator $T_{1}$ as follows:

$$
\left\|T_{1}\right\|:=\sup _{f(\neq \theta) \in L_{p, \Phi_{\delta}}\left(\mathbf{R}_{+}\right)} \frac{\left\|T_{1} f\right\|_{p, \widetilde{\Psi}^{1-p}}}{\|f\|_{p, \Phi_{\delta}}} .
$$

Then by [37, it is evident that $\left\|T_{1}\right\| \leq k(\sigma)$. Since by Theorem 2, the constant factor in (37) is the best possible, we have

$$
\left\|T_{1}\right\|=k(\sigma)=\frac{2 \Gamma\left(\frac{\sigma}{\gamma}\right)}{\gamma(2 \rho)^{\sigma / \gamma}} \zeta\left(\frac{\sigma}{\gamma}, \frac{\alpha+\rho}{2 \rho}\right) .
$$

Assuming that $a=\left\{a_{n}\right\}_{n=1}^{\infty} \in l_{q, \widetilde{\Psi}}$, setting

$$
h(x):=\sum_{n=1}^{\infty} \frac{\operatorname{csch}\left(\rho\left(U^{\delta}(x) \widetilde{V}_{n}\right)^{\gamma}\right)}{e^{\alpha\left(U^{\delta}(x) \widetilde{V}_{n}\right)^{\gamma}}} a_{n}, x \in \mathbf{R}_{+},
$$

we can rewrite (24) as follows: 


$$
\|h\|_{q, \Phi_{\delta}^{1-q}}<k(\sigma)\|a\|_{q, \widetilde{\Psi}}<\infty
$$

namely, $h \in L_{q, \Phi_{\delta}^{1-q}}\left(\mathbf{R}_{+}\right)$.

Definition 2. Define a half-discrete Hardy-Hilbert-type operator

$$
T_{2}: l_{q, \widetilde{\Psi}} \rightarrow L_{q, \Phi_{\delta}^{1-q}}\left(\mathbf{R}_{+}\right)
$$

as follows:

For any $a=\left\{a_{n}\right\}_{n=1}^{\infty} \in l_{q, \widetilde{\Psi}}$, there exists a unique representation $T_{2} a=h \in L_{q, \Phi_{\delta}^{1-q}}\left(\mathbf{R}_{+}\right)$. Define the formal inner product of $T_{2} a$ and $f \in L_{p, \Phi_{\delta}}\left(\mathbf{R}_{+}\right)$by:

$$
\left(T_{2} a, f\right):=\int_{0}^{\infty}\left[\sum_{n=1}^{\infty} \frac{\operatorname{csch}\left(\rho\left(U^{\delta}(x) \widetilde{V}_{n}\right)^{\gamma}\right)}{e^{\alpha\left(U^{\delta}(x) \widetilde{V}_{n}\right)^{\gamma}}} a_{n}\right] f(x) d x .
$$

Then we can rewrite (22) and (24) as follows:

$$
\begin{aligned}
\left(T_{2} a, f\right) & <k(\sigma)\|f\|_{p, \Phi_{\delta}}\|a\|_{q, \widetilde{\Psi}}, \\
\left\|T_{2} a\right\|_{q, \Phi_{\delta}^{1-q}} & <k(\sigma)\|a\|_{q, \widetilde{\Psi}} .
\end{aligned}
$$

Define the norm of operator $T_{2}$ by:

$$
\left\|T_{2}\right\|:=\sup _{a(\neq \theta) \in l_{q, \widetilde{\Psi}}} \frac{\left\|T_{2} a\right\|_{q, \Phi_{\delta}^{1-q}}}{\|a\|_{q, \widetilde{\Psi}}} .
$$

Then by 411, we find $\left\|T_{2}\right\| \leq k(\sigma)$. Since by Theorem 2, the constant factor in (41) is the best possible, we have

$$
\left\|T_{2}\right\|=k(\sigma)=\frac{2 \Gamma\left(\frac{\sigma}{\gamma}\right)}{\gamma(2 \rho)^{\sigma / \gamma}} \zeta\left(\frac{\sigma}{\gamma}, \frac{\alpha+\rho}{2 \rho}\right)=\left\|T_{1}\right\| .
$$

\section{Some Equivalent Reverses}

In the following, we also set

$$
\widetilde{\Phi}_{\delta}(x):=\left(1-\theta_{\delta}(\sigma, x)\right) \frac{U^{p(1-\delta \sigma)-1}(x)}{\mu^{p-1}(x)}\left(x \in \mathbf{R}_{+}\right) .
$$

For $0<p<1$ or $p<0$, we still use the formal symbols $\|f\|_{p, \Phi_{\delta}},\|f\|_{p, \widetilde{\Phi}_{\delta}}$ and $\|a\|_{q, \widetilde{\Psi}}$.

Theorem 3. If $0 \leq \alpha \leq \rho(\rho>0), 0<\gamma<\sigma \leq 1, k(\sigma)$ is given by (13), there exists $n_{0} \in \mathbf{N}$, such that $v_{n} \geq v_{n+1}\left(n \in\left\{n_{0}, n_{0}+1, \cdots\right\}\right)$, and $U(\infty)=V(\infty)=\infty$, then for 
$p<0,0<\|f\|_{p, \Phi_{\delta}},\|a\|_{q, \widetilde{\Psi}}<\infty$, we have the following equivalent inequalities with the best possible constant factor $k(\sigma)$ :

$$
\begin{aligned}
& I=\sum_{n=1}^{\infty} \int_{0}^{\infty} \frac{\operatorname{csch}\left(\rho\left(U^{\delta}(x) \widetilde{V}_{n}\right)^{\gamma}\right)}{e^{\alpha\left(U^{\delta}(x) \widetilde{V}_{n}\right)^{\gamma}}} a_{n} f(x) d x>k(\sigma)\|f\|_{p, \Phi_{\delta}}\|a\|_{q, \widetilde{\Psi}}, \\
& J_{1}=\sum_{n=1}^{\infty} \frac{v_{n}}{\widetilde{V}_{n}^{1-p \sigma}}\left[\int_{0}^{\infty} \frac{\operatorname{csch}\left(\rho\left(U^{\delta}(x) \widetilde{V}_{n}\right)^{\gamma}\right)}{e^{\alpha\left(U^{\delta}(x) \widetilde{V}_{n}\right)^{\gamma}}} f(x) d x\right]^{p}>k(\sigma)\|f\|_{p, \Phi_{\delta}}, \\
& J_{2}=\left\{\int_{0}^{\infty} \frac{\mu(x)}{U^{1-q \delta \sigma}(x)}\left[\sum_{n=1}^{\infty} \frac{\operatorname{csch}\left(\rho\left(U^{\delta}(x) \widetilde{V}_{n}\right)^{\gamma}\right)}{e^{\alpha\left(U^{\delta}(x) \widetilde{V}_{n}\right)^{\gamma}}} a_{n}\right]^{q} d x\right\}^{\frac{1}{q}} \\
& >k(\sigma)\|a\|_{q, \widetilde{\Psi}} \cdot
\end{aligned}
$$

Proof. By the reverse Hölder's inequality with weight (cf. [43]), since $p<0$, similarly to the way we obtained (25) and (26), we have

$$
\begin{aligned}
& {\left[\int_{0}^{\infty} \frac{\operatorname{csch}\left(\rho\left(U^{\delta}(x) \widetilde{V}_{n}\right)^{\gamma}\right)}{\left.e^{\alpha\left(U^{\delta}(x) \widetilde{V}_{n}\right)^{\gamma}} f(x) d x\right]^{p}}\right.} \\
\leq & \frac{\widetilde{V}_{n}^{1-p \sigma}}{\left(\varpi_{\delta}(\sigma, n)\right)^{1-p} v_{n}} \int_{0}^{\infty} \frac{\operatorname{csch}\left(\rho\left(U^{\delta}(x) \widetilde{V}_{n}\right)^{\gamma}\right)}{e^{\alpha\left(U^{\delta}(x) \widetilde{V}_{n}\right)^{\gamma}}} \frac{U^{(1-\delta \sigma)(p-1)}(x) v_{n}}{\widetilde{V}_{n}^{1-\sigma} \mu^{p-1}(x)} f^{p}(x) d x,
\end{aligned}
$$

and then by (19) and Lebesgue term by term integration theorem, it follows that

$$
\begin{aligned}
J_{1} & \geq(k(\sigma))^{\frac{1}{q}}\left[\sum_{n=1}^{\infty} \int_{0}^{\infty} \frac{\operatorname{csch}\left(\rho\left(U^{\delta}(x) \widetilde{V}_{n}\right)^{\gamma}\right)}{e^{\alpha\left(U^{\delta}(x) \widetilde{V}_{n}\right)^{\gamma}}} \frac{U^{(1-\delta \sigma)(p-1)}(x) v_{n}}{\widetilde{V}_{n}^{1-\sigma} \mu^{p-1}(x)} f^{p}(x) d x\right]^{\frac{1}{p}} \\
& =(k(\sigma))^{\frac{1}{q}}\left[\int_{0}^{\infty} \omega_{\delta}(\sigma, x) \frac{U^{p(1-\delta \sigma)-1}(x)}{\mu^{p-1}(x)} f^{p}(x) d x\right]^{\frac{1}{p}} .
\end{aligned}
$$

Then by (17), we have (44).

By the reverse Hölder's inequality (cf. [43]), we have

$$
\begin{aligned}
I & =\sum_{n=1}^{\infty}\left[\frac{v_{n}^{\frac{1}{p}}}{\widetilde{V}_{n}^{\frac{1}{p}-\sigma}} \int_{0}^{\infty} \frac{\operatorname{csch}\left(\rho\left(U^{\delta}(x) \widetilde{V}_{n}\right)^{\gamma}\right)}{e^{\alpha\left(U^{\delta}(x) \widetilde{V}_{n}\right)^{\gamma}}} f(x) d x\right]\left(\frac{\widetilde{V}_{n}^{\frac{1}{p}-\sigma} a_{n}}{v_{n}^{\frac{1}{p}}}\right) \\
& \geq J_{1}\|a\|_{q, \widetilde{\Psi}}
\end{aligned}
$$

Then by (44), we have (43).

On the other hand, assuming that (43) is valid, we set $a_{n}$ as in Theorem 1. Then we find $J_{1}^{p}=\|a\|_{q, \widetilde{\Psi}}^{q}$.

If $J_{1}=\infty$, then (44) is trivially valid.

If $J_{1}=0$, then (44) is impossible.

Suppose that $0<J_{1}<\infty$. By [43], it follows that 


$$
\begin{aligned}
& \|a\|_{q, \widetilde{\Psi}}^{q}=J_{1}^{p}=I>k(\sigma)\|f\|_{p, \Phi_{\delta}}\|a\|_{q, \widetilde{\Psi}}, \\
& \|a\|_{q, \widetilde{\Psi}}^{q-1}=J_{1}>k(\sigma)\|f\|_{p, \Phi_{\delta}},
\end{aligned}
$$

and then (44) follows, which is equivalent to (43).

By the reverse of Hölder's inequality with weight (cf. [43]), since $0<q<1$, similarly to the way we obtained (28) and (29), we have

$$
\begin{aligned}
& {\left[\sum_{n=1}^{\infty} \frac{\operatorname{csch}\left(\rho\left(U^{\delta}(x) \widetilde{V}_{n}\right)^{\gamma}\right)}{e^{\alpha\left(U^{\delta}(x) \widetilde{V}_{n}\right)^{\gamma}}} a_{n}\right]^{q}} \\
& \geq \frac{\left(\omega_{\delta}(\sigma, x)\right)^{q-1}}{U^{q \delta \sigma-1}(x) \mu(x)} \sum_{n=1}^{\infty} \frac{\operatorname{csch}\left(\rho\left(U^{\delta}(x) \widetilde{V}_{n}\right)^{\gamma}\right)}{e^{\alpha\left(U^{\delta}(x) \widetilde{V}_{n}\right)^{\gamma}}} \frac{\widetilde{V}_{n}^{(1-\sigma)(q-1)} \mu(x)}{U^{1-\delta \sigma}(x) v_{n}^{q-1}} a_{n}^{q},
\end{aligned}
$$

and then by (17) and Lebesgue term by term integration theorem, it follows that

$$
\begin{aligned}
J_{2} & >(k(\sigma))^{\frac{1}{p}}\left[\int_{0}^{\infty} \sum_{n=1}^{\infty} \frac{\operatorname{csch}\left(\rho\left(U^{\delta}(x) \widetilde{V}_{n}\right)^{\gamma}\right)}{e^{\alpha\left(U^{\delta}(x) \widetilde{V}_{n}\right)^{\gamma}}} \frac{\widetilde{V}_{n}^{(1-\sigma)(q-1)} \mu(x)}{U^{1-\delta \sigma}(x) v_{n}^{q-1}} a_{n}^{q} d x\right]^{\frac{1}{q}} \\
& =(k(\sigma))^{\frac{1}{p}}\left[\sum_{n=1}^{\infty} \varpi_{\delta}(\sigma, n) \frac{\widetilde{V}_{n}^{q(1-\sigma)-1}}{v_{n}^{q-1}} a_{n}^{q}\right]^{\frac{1}{q}} .
\end{aligned}
$$

Then by 197, we obtain 45).

By the reverse Hölder's inequality (cf. [43]), we get

$$
\begin{aligned}
I & =\int_{0}^{\infty}\left(\frac{U^{\frac{1}{q}-\delta \sigma}(x)}{\mu^{\frac{1}{q}}(x)} f(x)\right)\left[\frac{\mu^{\frac{1}{q}}(x)}{U^{\frac{1}{q}-\delta \sigma}(x)} \sum_{n=1}^{\infty} \frac{\operatorname{csch}\left(\rho\left(U^{\delta}(x) \widetilde{V}_{n}\right)^{\gamma}\right)}{e^{\alpha\left(U^{\delta}(x) \widetilde{V}_{n}\right)^{\gamma}}} a_{n}\right] d x \\
& \geq\|f\|_{p, \Phi_{\delta}} J_{2} .
\end{aligned}
$$

Then by (45), we derive 433).

On the other hand, assuming that (45) is valid, we set $f(x)$ as in Theorem 1. Then we find $J_{2}^{q}=\|f\|_{p, \Phi_{\delta}}^{p}$.

If $J_{2}=\infty$, then (45) is trivially valid.

If $J_{2}=0$, then (45) keeps impossible.

Suppose that $0<J_{2}<\infty$. By [43], it follows that

$$
\begin{aligned}
& \|f\|_{p, \Phi_{\delta}}^{p}=J_{2}^{q}=I>k(\sigma)\|f\|_{p, \Phi_{\delta}}\|a\|_{q, \widetilde{\Psi}}, \\
& \|f\|_{p, \Phi_{\delta}}^{p-1}=J_{2}>k(\sigma)\|a\|_{q, \widetilde{\Psi}},
\end{aligned}
$$

and then (45) follows, which is equivalent to (43).

Therefore, inequalities (43), (44) and (45) are equivalent.

For $\varepsilon \in(0, q(\sigma-\gamma))$, we set $\widetilde{\sigma}=\sigma-\frac{\varepsilon}{q}(\in(\gamma, 1))$, and $\widetilde{f}=\widetilde{f}(x), x \in \mathbf{R}_{+}, \widetilde{a}=$ $\left\{\widetilde{a}_{n}\right\}_{n=1}^{\infty}$, 


$$
\begin{gathered}
\widetilde{f}(x)=\left\{\begin{array}{c}
U^{\delta(\widetilde{\sigma}+\varepsilon)-1}(x) \mu(x), 0<x^{\delta} \leq 1 \\
0, x^{\delta}>0
\end{array}\right. \\
\widetilde{a}_{n}=\widetilde{V}_{n}^{\widetilde{\sigma}-1} v_{n}=\widetilde{V}_{n}^{\sigma-\frac{\varepsilon}{q}-1} v_{n}, n \in \mathbf{N} .
\end{gathered}
$$

By 21), 33) and 17), we obtain

$$
\begin{gathered}
\|\widetilde{f}\|_{p, \Phi_{\delta}}\|\widetilde{a}\|_{q, \widetilde{\Psi}}=\frac{1}{\varepsilon} U^{\frac{\delta \varepsilon}{p}}(1)\left(\frac{1}{V_{n_{0}}^{\varepsilon}}+\varepsilon \widetilde{O}(1)\right)^{\frac{1}{q}}, \\
\widetilde{I}=\sum_{n=1}^{\infty} \int_{0}^{\infty} \frac{\operatorname{csch}\left(\rho\left(U^{\delta}(x) \widetilde{V}_{n}\right)^{\gamma}\right)}{e^{\alpha\left(U^{\delta}(x) \widetilde{V}_{n}\right)^{\gamma}}} \widetilde{a}_{n} \widetilde{f}(x) d x \\
=\int_{\left\{x>0 ; 0<x^{\delta} \leq 1\right\}} \omega_{\delta}(\widetilde{\sigma}, x) \frac{\mu(x)}{U^{1-\delta \varepsilon}(x)} d x \\
\leq k(\widetilde{\sigma}) \int_{\left\{x>0 ; 0<x^{\delta} \leq 1\right\}} \frac{\mu(x)}{U^{1-\delta \varepsilon}(x)} d x=\frac{1}{\varepsilon} k\left(\sigma-\frac{\varepsilon}{q}\right) U^{\delta \varepsilon}(1) .
\end{gathered}
$$

If there exists a positive constant $K \geq k(\sigma)$, such that (43) is valid when replacing $k(\sigma)$ by $K$, then in particular, we have $\varepsilon \widetilde{I}>\varepsilon K\|\widetilde{f}\|_{p, \Phi_{\delta}}\|\widetilde{a}\|_{q, \widetilde{\Psi}}$, namely,

$$
k\left(\sigma-\frac{\varepsilon}{q}\right) U^{\delta \varepsilon}(1)>K \cdot U^{\frac{\delta \varepsilon}{p}}(1)\left(\frac{1}{V_{n_{0}}^{\varepsilon}}+\varepsilon \widetilde{O}(1)\right)^{\frac{1}{q}} .
$$

It follows that $k(\sigma) \geq K\left(\varepsilon \rightarrow 0^{+}\right)$. Hence, $K=k(\sigma)$ is the best possible constant factor of 43.

The constant factor $k(\sigma)$ in (44) (45)) is still the best possible. Otherwise, we would reach a contradiction by (46) (477) that the constant factor in 43) is not the best possible. The theorem is proved.

Theorem 4. With the assumptions of Theorem 3, if

$$
0<p<1,0<\|f\|_{p, \Phi_{\delta}},\|a\|_{q, \widetilde{\Psi}}<\infty
$$

then we have the following equivalent inequalities with the best possible constant factor $k(\sigma)$ : 


$$
\begin{aligned}
I & =\sum_{n=1}^{\infty} \int_{0}^{\infty} \frac{\operatorname{csch}\left(\rho\left(U^{\delta}(x) \widetilde{V}_{n}\right)^{\gamma}\right)}{e^{\alpha\left(U^{\delta}(x) \widetilde{V}_{n}\right)^{\gamma}}} a_{n} f(x) d x>k(\sigma)\|f\|_{p, \widetilde{\Phi}_{\delta}}\|a\|_{q, \widetilde{\Psi}}, \\
J_{1} & =\sum_{n=1}^{\infty} \frac{v_{n}}{\widetilde{V}_{n}^{1-p \sigma}}\left[\int_{0}^{\infty} \frac{\operatorname{csch}\left(\rho\left(U^{\delta}(x) \widetilde{V}_{n}\right)^{\gamma}\right)}{e^{\alpha\left(U^{\delta}(x) \widetilde{V}_{n}\right)^{\gamma}}} f(x) d x\right]^{p}>k(\sigma)\|f\|_{p, \widetilde{\Phi}_{\delta}} \\
J & :=\left\{\int_{0}^{\infty} \frac{\left(1-\theta_{\delta}(\sigma, x)\right)^{1-q} \mu(x)}{U^{1-q \delta \sigma}(x)}\left[\sum_{n=1}^{\infty} \frac{\operatorname{csch}\left(\rho\left(U^{\delta}(x) \widetilde{V}_{n}\right)^{\gamma}\right)}{e^{\alpha\left(U^{\delta}(x) \widetilde{V}_{n}\right)^{\gamma}}} a_{n}\right]^{q} d x\right\}^{\frac{1}{q}} \\
& >k(\sigma)\|a\|_{q, \widetilde{\Psi}} .
\end{aligned}
$$

Proof. By the reverse Hölder's inequality with weight (cf. [43]), since $0<p<1$, similarly to the way we obtained (25) and (26), we have

$$
\begin{aligned}
& {\left[\int_{0}^{\infty} \frac{\operatorname{csch}\left(\rho\left(U^{\delta}(x) \widetilde{V}_{n}\right)^{\gamma}\right)}{e^{\alpha\left(U^{\delta}(x) \widetilde{V}_{n}\right)^{\gamma}}} f(x) d x\right]^{p}} \\
& \geq \frac{\left(\varpi_{\delta}(\sigma, n)\right)^{p-1}}{\widetilde{V}_{n}^{p \sigma-1} v_{n}} \int_{0}^{\infty} \frac{\operatorname{csch}\left(\rho\left(U^{\delta}(x) \widetilde{V}_{n}\right)^{\gamma}\right)}{e^{\alpha\left(U^{\delta}(x) \widetilde{V}_{n}\right)^{\gamma}}} \frac{U^{(1-\delta \sigma)(p-1)}(x) v_{n}}{\widetilde{V}_{n}^{1-\sigma} \mu^{p-1}(x)} f^{p}(x) d x,
\end{aligned}
$$

and then in view of (19) and Lebesgue term by term integration theorem, we find

$$
\begin{aligned}
J_{1} & \geq(k(\sigma))^{\frac{1}{q}}\left[\sum_{n=1}^{\infty} \int_{0}^{\infty} \frac{\operatorname{csch}\left(\rho\left(U^{\delta}(x) \widetilde{V}_{n}\right)^{\gamma}\right)}{e^{\alpha\left(U^{\delta}(x) \widetilde{V}_{n}\right)^{\gamma}}} \frac{U^{(1-\delta \sigma)(p-1)}(x) v_{n}}{\widetilde{V}_{n}^{1-\sigma} \mu^{p-1}(x)} f^{p}(x) d x\right]^{\frac{1}{p}} \\
& =(k(\sigma))^{\frac{1}{q}}\left[\int_{0}^{\infty} \omega_{\delta}(\sigma, x) \frac{U^{p(1-\delta \sigma)-1}(x)}{\mu^{p-1}(x)} f^{p}(x) d x\right]^{\frac{1}{p}} .
\end{aligned}
$$

Then by 20, we have 50.

By the reverse Hölder's inequality (cf. [43]), we have

$$
\begin{aligned}
I & =\sum_{n=1}^{\infty}\left[\frac{v_{n}^{\frac{1}{p}}}{\widetilde{V}_{n}^{\frac{1}{p}-\sigma}} \int_{0}^{\infty} \frac{\operatorname{csch}\left(\rho\left(U^{\delta}(x) \widetilde{V}_{n}\right)^{\gamma}\right)}{e^{\alpha\left(U^{\delta}(x) \widetilde{V}_{n}\right)^{\gamma}}} f(x) d x\right]\left(\frac{\widetilde{V}_{n}^{\frac{1}{p}-\sigma} a_{n}}{v_{n}^{\frac{1}{p}}}\right) \\
& \geq J_{1}\|a\|_{q, \widetilde{\Psi}}
\end{aligned}
$$

Then by (50), we have 49.)

On the other hand, Assuming that 49) is valid, we set $a_{n}$ as in Theorem 1. Then we find $J_{1}^{p}=\|a\|_{q}^{q} \widetilde{\Psi}$.

If $J_{1}=\infty$, then (50) is trivially valid.

If $J_{1}=0$, then (50) remains impossible.

Suppose that $0<J_{1}<\infty$. By [49, it follows that 


$$
\begin{aligned}
& \|a\|_{q, \widetilde{\Psi}}^{q}=J_{1}^{p}=I>k(\sigma)\|f\|_{p, \widetilde{\Phi}_{\delta}}\|a\|_{q, \widetilde{\Psi}}, \\
& \|a\|_{q, \widetilde{\Psi}}^{q-1}=J_{1}>k(\sigma)\|f\|_{p, \widetilde{\Phi}_{\delta}},
\end{aligned}
$$

and then (50) follows, which is equivalent to (49).

By the reverse Hölder's inequality with weight (cf. [43]), since $q<0$, we have

$$
\begin{aligned}
& {\left[\sum_{n=1}^{\infty} \frac{\operatorname{csch}\left(\rho\left(U^{\delta}(x) \widetilde{V}_{n}\right)^{\gamma}\right)}{e^{\alpha\left(U^{\delta}(x) \widetilde{V}_{n}\right)^{\gamma}}} a_{n}\right]^{q}} \\
& \leq \frac{\left(\omega_{\delta}(\sigma, x)\right)^{q-1}}{U^{q \delta \sigma-1}(x) \mu(x)} \sum_{n=1}^{\infty} \frac{\operatorname{csch}\left(\rho\left(U^{\delta}(x) \widetilde{V}_{n}\right)^{\gamma}\right)}{e^{\alpha\left(U^{\delta}(x) \widetilde{V}_{n}\right)^{\gamma}}} \frac{\widetilde{V}_{n}^{(1-\sigma)(q-1)} \mu(x)}{U^{1-\delta \sigma}(x) v_{n}^{q-1}} a_{n}^{q},
\end{aligned}
$$

and then by 20) and Lebesgue term by term integration theorem, it follows that

$$
\begin{aligned}
J & >(k(\sigma))^{\frac{1}{p}}\left[\int_{0}^{\infty} \sum_{n=1}^{\infty} \frac{\operatorname{csch}\left(\rho\left(U^{\delta}(x) \widetilde{V}_{n}\right)^{\gamma}\right)}{e^{\alpha\left(U^{\delta}(x) \widetilde{V}_{n}\right)^{\gamma}}} \frac{\widetilde{V}_{n}^{(1-\sigma)(q-1)} \mu(x)}{U^{1-\delta \sigma}(x) v_{n}^{q-1}} a_{n}^{q} d x\right]^{\frac{1}{q}} \\
& =(k(\sigma))^{\frac{1}{p}}\left[\sum_{n=1}^{\infty} \varpi_{\delta}(\sigma, n) \frac{\widetilde{V}_{n}^{q(1-\sigma)-1}}{v_{n}^{q-1}} a_{n}^{q}\right]^{\frac{1}{q}} .
\end{aligned}
$$

Then by (19), we have (51).

By the reverse Hölder's inequality (cf. [43]), we have

$$
\begin{aligned}
I= & \int_{0}^{\infty}\left[\left(1-\theta_{\delta}(\sigma, x)\right)^{\frac{1}{p}} \frac{U^{\frac{1}{q}-\delta \sigma}(x)}{\mu^{\frac{1}{q}}(x)} f(x)\right] \\
& \times\left[\frac{\left(1-\theta_{\delta}(\sigma, x)\right)^{\frac{-1}{p}} \mu^{\frac{1}{q}}(x)}{U^{\frac{1}{q}-\delta \sigma}(x)} \sum_{n=1}^{\infty} \frac{\operatorname{csch}\left(\rho\left(U^{\delta}(x) \widetilde{V}_{n}\right)^{\gamma}\right)}{e^{\alpha\left(U^{\delta}(x) \widetilde{V}_{n}\right)^{\gamma}}} a_{n}\right] d x \\
\geq & \|f\|_{p, \widetilde{\Phi}_{\delta} J .}
\end{aligned}
$$

Then by (51), we have 499).

On the other hand, assuming that (49) is valid, we set $f(x)$ as in Theorem 1. Then we find $J^{q}=\|f\|_{p}^{p} \widetilde{\Phi}_{\delta}$.

If $J=\infty$, then (51) is trivially valid.

If $J=0$, then (51) remains impossible.

Suppose that $0<J<\infty$. By (49), it follows that

$$
\begin{aligned}
& \|f\|_{p, \widetilde{\Phi}_{\delta}}^{p}=J^{q}=I>k(\sigma)\|f\|_{p, \widetilde{\Phi}_{\delta}}\|a\|_{q, \widetilde{\Psi}}, \\
& \|f\|_{p, \widetilde{\Phi}_{\delta}}^{p-1}=J>k(\sigma)\|a\|_{q, \widetilde{\Psi}}
\end{aligned}
$$

and then (51) follows, which is equivalent to 497.

Therefore, inequalities (49), (50) and 511) are equivalent. 
For $\varepsilon \in(0, p(\sigma-\gamma))$, we set $\widetilde{\sigma}=\sigma+\frac{\varepsilon}{p}(>\gamma)$, and $\widetilde{f}=\widetilde{f}(x), x \in \mathbf{R}_{+}, \widetilde{a}=\left\{\widetilde{a}_{n}\right\}_{n=1}^{\infty}$,

$$
\begin{aligned}
\widetilde{f}(x) & =\left\{\begin{array}{c}
U^{\delta \widetilde{\sigma}-1}(x) \mu(x), 0<x^{\delta} \leq 1 \\
0, x^{\delta}>0
\end{array}\right. \\
\widetilde{a}_{n} & =\widetilde{V}_{n}^{\widetilde{\sigma}-\varepsilon-1} v_{n}=\widetilde{V}_{n}^{\sigma-\frac{\varepsilon}{q}-1} v_{n}, n \in \mathbf{N} .
\end{aligned}
$$

By (20), 21) and (33), we obtain

$$
\begin{aligned}
& \left\|\left.\widetilde{f}\right|_{p, \widetilde{\Phi}_{\delta}}\right\| \widetilde{a} \|_{q, \widetilde{\Psi}} \\
& =\left[\int_{\left\{x>0 ; 0<x^{\delta} \leq 1\right\}}\left(1-O\left((U(x))^{\delta(\sigma-\gamma)}\right)\right) \frac{\mu(x) d x}{U^{1-\delta \varepsilon}(x)}\right]^{\frac{1}{p}}\left(\sum_{n=1}^{\infty} \frac{v_{n}}{\widetilde{V}_{n}^{1+\varepsilon}}\right)^{\frac{1}{q}} \\
& =\frac{1}{\varepsilon}\left(U^{\delta \varepsilon}(1)-\varepsilon O(1)\right)^{\frac{1}{p}}\left(\frac{1}{V_{n_{0}}^{\varepsilon}}+\varepsilon \widetilde{O}(1)\right)^{\frac{1}{q}} \\
& \widetilde{I}=\sum_{n=1}^{\infty} \int_{0}^{\infty} \frac{\operatorname{csch}\left(\rho\left(U^{\delta}(x) \widetilde{V}_{n}\right)^{\gamma}\right)}{e^{\alpha\left(U^{\delta}(x) \widetilde{V}_{n}\right)^{\gamma}}} \widetilde{a}_{n} \widetilde{f}(x) d x \\
& =\sum_{n=1}^{\infty}\left[\int_{\left\{x>0 ; 0<x^{\delta} \leq 1\right\}} \frac{\operatorname{csch}\left(\rho\left(U^{\delta}(x) \widetilde{V}_{n}\right)^{\gamma}\right)}{e^{\alpha\left(U^{\delta}(x) \widetilde{V}_{n}\right)^{\gamma}}} \frac{\widetilde{V}_{n}^{\widetilde{\sigma}} \mu(x)}{U^{1-\delta \widetilde{\sigma}(x)}} d x\right] \frac{v_{n}}{\widetilde{V}_{n}^{1+\varepsilon}} \\
& \leq \sum_{n=1}^{\infty}\left[\int_{0}^{\infty} \frac{\operatorname{csch}\left(\rho\left(U^{\delta}(x) \widetilde{V}_{n}\right)^{\gamma}\right)}{e^{\alpha\left(U^{\delta}(x) \widetilde{V}_{n}\right)^{\gamma}}} \frac{\widetilde{V}_{n}^{\widetilde{\sigma}} \mu(x)}{U^{1-\delta \widetilde{\sigma}}(x)} d x\right] \frac{v_{n}}{\widetilde{V}_{n}^{1+\varepsilon}} \\
& =\sum_{n=1}^{\infty} \varpi_{\delta}(\widetilde{\sigma}, n) \frac{v_{n}}{\widetilde{V}_{n}^{1+\varepsilon}}=k(\widetilde{\sigma}) \sum_{n=1}^{\infty} \frac{v_{n}}{\widetilde{V}_{n}^{1+\varepsilon}} \\
& =\frac{1}{\varepsilon} k\left(\sigma+\frac{\varepsilon}{p}\right)\left(\frac{1}{V_{n_{0}}^{\varepsilon}}+\varepsilon \widetilde{O}(1)\right) \text {. }
\end{aligned}
$$

If there exists a positive constant $K \geq k(\sigma)$, such that (43) is valid when replacing $k(\sigma)$ by $K$, then in particular, we have $\varepsilon \widetilde{I}>\varepsilon K|| \widetilde{f}\left\|_{p, \widetilde{\Phi}_{\delta}}|| \widetilde{a}\right\|_{q, \widetilde{\Psi}}$, namely,

$$
\begin{aligned}
& k\left(\sigma+\frac{\varepsilon}{p}\right)\left(\frac{1}{V_{n_{0}}^{\varepsilon}}+\varepsilon \widetilde{O}(1)\right) \\
> & K\left(U^{\delta \varepsilon}(1)-\varepsilon O(1)\right)^{\frac{1}{p}}\left(\frac{1}{V_{n_{0}}^{\varepsilon}}+\varepsilon \widetilde{O}(1)\right)^{\frac{1}{q}} .
\end{aligned}
$$

It follows that $k(\sigma) \geq K\left(\varepsilon \rightarrow 0^{+}\right)$. Hence, $K=k(\sigma)$ is the best possible constant factor of 49]. 
The constant factor $k(\sigma)$ in (50) (51) $)$ is still the best possible. Otherwise, we would reach a contradiction by (52) (53) that the constant factor in $(49)$ is not the best possible. The theorem is proved.

\section{Some Particular Inequalities}

For $\widetilde{v}_{n}=0, \widetilde{V}_{n}=V_{n}$, we set

$$
\Psi(n):=\frac{V_{n}^{q(1-\sigma)-1}}{v_{n}^{q-1}}(n \in \mathbf{N}) .
$$

In view of Theorem 2-4, we have

Corollary 1. If $0 \leq \alpha \leq \rho(\rho>0), 0<\gamma<\sigma \leq 1, k(\sigma)$ is given by (13), there exists $n_{0} \in \mathbf{N}$, such that $v_{n} \geq v_{n+1}\left(n \in\left\{n_{0}, n_{0}+1, \cdots\right\}\right)$, and $U(\infty)=V(\infty)=\infty$, then (i) for $p>1,0<\|f\|_{p, \Phi_{\delta}},\|a\|_{q, \Psi}<\infty$, we have the following equivalent inequalities:

$$
\begin{gathered}
\sum_{n=1}^{\infty} \int_{0}^{\infty} \frac{\operatorname{csch}\left(\rho\left(U^{\delta}(x) V_{n}\right)^{\gamma}\right)}{e^{\alpha\left(U^{\delta}(x) V_{n}\right)^{\gamma}} a_{n} f(x) d x<k(\sigma)\|f\|_{p, \Phi_{\delta}}\|a\|_{q, \Psi},} \\
\sum_{n=1}^{\infty} \frac{v_{n}}{V_{n}^{1-p \sigma}}\left[\int_{0}^{\infty} \frac{\operatorname{csch}\left(\rho\left(U^{\delta}(x) V_{n}\right)^{\gamma}\right)}{\left.e^{\alpha\left(U^{\delta}(x) V_{n}\right)^{\gamma}} f(x) d x\right]^{p}<k(\sigma)\|f\|_{p, \Phi_{\delta},}}\right. \\
\left\{\int_{0}^{\infty} \frac{\mu(x)}{U^{1-q \delta \sigma}(x)}\left[\sum_{n=1}^{\infty} \frac{\operatorname{csch}\left(\rho\left(U^{\delta}(x) V_{n}\right)^{\gamma}\right)}{e^{\alpha\left(U^{\delta}(x) V_{n}\right)^{\gamma}}} a_{n}\right]^{q} d x\right\}^{\frac{1}{q}}<k(\sigma)\|a\|_{q, \Psi} ;
\end{gathered}
$$

(ii) for $p<0,0<\|f\|_{p, \Phi_{\delta}},\|a\|_{q, \Psi}<\infty$, we have the following equivalent inequalities:

$$
\begin{gathered}
\sum_{n=1}^{\infty} \int_{0}^{\infty} \frac{\operatorname{csch}\left(\rho\left(U^{\delta}(x) V_{n}\right)^{\gamma}\right)}{e^{\alpha\left(U^{\delta}(x) V_{n}\right)^{\gamma}} a_{n} f(x) d x>k(\sigma)\|f\|_{p, \Phi_{\delta}}\|a\|_{q, \Psi},} \\
\sum_{n=1}^{\infty} \frac{v_{n}}{V_{n}^{1-p \sigma}}\left[\int_{0}^{\infty} \frac{\operatorname{csch}\left(\rho\left(U^{\delta}(x) V_{n}\right)^{\gamma}\right)}{\left.e^{\alpha\left(U^{\delta}(x) V_{n}\right)^{\gamma}} f(x) d x\right]^{p}>k(\sigma)\|f\|_{p, \Phi_{\delta},}}\right. \\
\left\{\int_{0}^{\infty} \frac{\mu(x)}{U^{1-q \delta \sigma}(x)}\left[\sum_{n=1}^{\infty} \frac{\operatorname{csch}\left(\rho\left(U^{\delta}(x) V_{n}\right)^{\gamma}\right)}{e^{\alpha\left(U^{\delta}(x) V_{n}\right)^{\gamma}}} a_{n}\right]^{q} d x\right\}^{\frac{1}{q}}>k(\sigma)\|a\|_{q, \Psi} ;
\end{gathered}
$$

(iii) for $0<p<1,0<\|f\|_{p, \Phi_{\delta}},\|a\|_{q, \Psi}<\infty$, we have the following equivalent inequalities: 


$$
\begin{aligned}
& \sum_{n=1}^{\infty} \int_{0}^{\infty} \frac{\csc h\left(\rho\left(U^{\delta}(x) V_{n}\right)^{\gamma}\right)}{e^{\alpha\left(U^{\delta}(x) V_{n}\right)^{\gamma}}} a_{n} f(x) d x>k(\sigma)\|f\|_{p, \widetilde{\Phi}_{\delta}}\|a\|_{q, \Psi}, \\
& \sum_{n=1}^{\infty} \frac{v_{n}}{V_{n}^{1-p \sigma}}\left[\int_{0}^{\infty} \frac{\operatorname{csch}\left(\rho\left(U^{\delta}(x) V_{n}\right)^{\gamma}\right)}{e^{\alpha\left(U^{\delta}(x) V_{n}\right)^{\gamma}}} f(x) d x\right]^{p}>k(\sigma)\|f\|_{p, \widetilde{\Phi}_{\delta}}, \\
& \left\{\int_{0}^{\infty} \frac{\left(1-\theta_{\delta}(\sigma, x)\right)^{1-q} \mu(x)}{U^{1-q \delta \sigma}(x)}\left[\sum_{n=1}^{\infty} \frac{\operatorname{csch}\left(\rho\left(U^{\delta}(x) V_{n}\right)^{\gamma}\right)}{e^{\alpha\left(U^{\delta}(x) V_{n}\right)^{\gamma}}} a_{n}\right]^{q} d x\right\}^{\frac{1}{q}} \\
& >k(\sigma)\|a\|_{q, \Psi} \text {. }
\end{aligned}
$$

The above inequalities have the best possible constant factor $k(\sigma)$.

In particular, for $\delta=1$, we have the following inequalities with the non-homogeneous kernel:

Corollary 2. If $0 \leq \alpha \leq \rho(\rho>0), 0<\gamma<\sigma \leq 1, k(\sigma)$ is given by (13), there exists $n_{0} \in \mathbf{N}$, such that $v_{n} \geq v_{n+1}\left(n \in\left\{n_{0}, n_{0}+1, \cdots\right\}\right)$, and $U(\infty)=V(\infty)=\infty$, then (i) for $p>1,0<\|f\|_{p, \Phi_{1}},\|a\|_{q, \Psi}<\infty$, we have the following equivalent inequalities:

$$
\begin{aligned}
& \sum_{n=1}^{\infty} \int_{0}^{\infty} \frac{\operatorname{csch}\left(\rho\left(U(x) V_{n}\right)^{\gamma}\right)}{e^{\alpha\left(U(x) V_{n}\right)^{\gamma}}} a_{n} f(x) d x<k(\sigma)\|f\|_{p, \Phi_{1}}\|a\|_{q, \Psi}, \\
& \sum_{n=1}^{\infty} \frac{v_{n}}{V_{n}^{1-p \sigma}}\left[\int_{0}^{\infty} \frac{\operatorname{csch}\left(\rho\left(U(x) V_{n}\right)^{\gamma}\right)}{e^{\alpha\left(U(x) V_{n}\right)^{\gamma}}} f(x) d x\right]^{p}<k(\sigma)\|f\|_{p, \Phi_{1}}, \\
& \left\{\int_{0}^{\infty} \frac{\mu(x)}{U^{1-q \sigma}(x)}\left[\sum_{n=1}^{\infty} \frac{\operatorname{csch}\left(\rho\left(U(x) V_{n}\right)^{\gamma}\right)}{e^{\alpha\left(U(x) V_{n}\right)^{\gamma}}} a_{n}\right]^{q} d x\right\}^{\frac{1}{q}}<k(\sigma)\|a\|_{q, \Psi}
\end{aligned}
$$

(ii) for $p<0,0<\|f\|_{p, \Phi_{1}},\|a\|_{q, \Psi}<\infty$, we have the following equivalent inequalities:

$$
\begin{gathered}
\sum_{n=1}^{\infty} \int_{0}^{\infty} \frac{\operatorname{csch}\left(\rho\left(U(x) V_{n}\right)^{\gamma}\right)}{e^{\alpha\left(U(x) V_{n}\right)^{\gamma}} a_{n} f(x) d x>k(\sigma)\|f\|_{p, \Phi_{1}}\|a\|_{q, \Psi},} \\
\sum_{n=1}^{\infty} \frac{v_{n}}{V_{n}^{1-p \sigma}}\left[\int_{0}^{\infty} \frac{\operatorname{csch}\left(\rho\left(U(x) V_{n}\right)^{\gamma}\right)}{\left.e^{\alpha\left(U(x) V_{n}\right)^{\gamma}} f(x) d x\right]^{p}>k(\sigma)\|f\|_{p, \Phi_{1}},}\right. \\
\left\{\int_{0}^{\infty} \frac{\mu(x)}{U^{1-q \sigma}(x)}\left[\sum_{n=1}^{\infty} \frac{\operatorname{csch}\left(\rho\left(U(x) V_{n}\right)^{\gamma}\right)}{e^{\alpha\left(U(x) V_{n}\right)^{\gamma}}} a_{n}\right]^{q} d x\right\}^{\frac{1}{q}}>k(\sigma)\|a\|_{q, \Psi}
\end{gathered}
$$

(iii) for $0<p<1,0<\|f\|_{p, \Phi_{1}},\|a\|_{q, \Psi}<\infty$, we have the following equivalent inequalities: 


$$
\begin{aligned}
& \sum_{n=1}^{\infty} \int_{0}^{\infty} \frac{\operatorname{csch}\left(\rho\left(U(x) V_{n}\right)^{\gamma}\right)}{e^{\alpha\left(U(x) V_{n}\right)^{\gamma}}} a_{n} f(x) d x>k(\sigma)\|f\|_{p, \widetilde{\Phi}_{1}}\|a\|_{q, \Psi}, \\
& \sum_{n=1}^{\infty} \frac{v_{n}}{V_{n}^{1-p \sigma}}\left[\int_{0}^{\infty} \frac{\operatorname{csch}\left(\rho\left(U(x) V_{n}\right)^{\gamma}\right)}{e^{\alpha\left(U(x) V_{n}\right)^{\gamma}}} f(x) d x\right]^{p}>k(\sigma)\|f\|_{p, \widetilde{\Phi}_{1},} \\
& \left\{\int_{0}^{\infty} \frac{\left(1-\theta_{1}(\sigma, x)\right)^{1-q} \mu(x)}{U^{1-q \sigma}(x)}\left[\sum_{n=1}^{\infty} \frac{\operatorname{csch}\left(\rho\left(U(x) V_{n}\right)^{\gamma}\right)}{e^{\alpha\left(U(x) V_{n}\right)^{\gamma}}} a_{n}\right]^{q} d x\right\}^{\frac{1}{q}} \\
> & k(\sigma)\|a\|_{q, \Psi} .
\end{aligned}
$$

The above inequalities involve the best possible constant factor $k(\sigma)$.

For $\delta=-1$, we have the following inequalities with the homogeneous kernel of degree 0 :

Corollary 3. If $0 \leq \alpha \leq \rho(\rho>0), 0<\gamma<\sigma \leq 1, k(\sigma)$ is given by (13), there exists $n_{0} \in \mathbf{N}$, such that $v_{n} \geq v_{n+1}\left(n \in\left\{n_{0}, n_{0}+1, \cdots\right\}\right)$, and $U(\infty)=V(\infty)=\infty$, then (i) for $p>1,0<\|f\|_{p, \Phi_{-1}},\|a\|_{q, \Psi}<\infty$, we have the following equivalent inequalities:

$$
\begin{gathered}
\sum_{n=1}^{\infty} \int_{0}^{\infty} \frac{\operatorname{csch}\left(\rho\left(\frac{V_{n}}{U(x)}\right)^{\gamma}\right)}{e^{\alpha\left(\frac{V_{n}}{U(x)}\right)^{\gamma}} a_{n} f(x) d x<k(\sigma)\|f\|_{p, \Phi_{-1}}\|a\|_{q, \Psi},} \\
\sum_{n=1}^{\infty} \frac{v_{n}}{V_{n}^{1-p \sigma}}\left[\int_{0}^{\infty} \frac{\operatorname{csch}\left(\rho\left(\frac{V_{n}}{U(x)}\right)^{\gamma}\right)}{\left.e^{\alpha\left(\frac{V_{n}}{U(x)}\right)^{\gamma}} f(x) d x\right]^{p}<k(\sigma)\|f\|_{p, \Phi_{-1}},}\right. \\
\left\{\int _ { 0 } ^ { \infty } \frac { \mu ( x ) } { U ^ { 1 + q \sigma } ( x ) } \left[\sum_{n=1}^{\infty} \frac{\operatorname{csch}\left(\rho\left(\frac{V_{n}}{U(x)}\right)^{\gamma}\right)}{\left.\left.e^{\alpha\left(\frac{V_{n}}{U(x)}\right)^{\gamma}} a_{n}\right]^{q} d x\right\}^{\frac{1}{q}}<k(\sigma)\|a\|_{q, \Psi} ;}\right.\right.
\end{gathered}
$$

(ii) for $p<0,0<\|f\|_{p, \Phi_{-1}},\|a\|_{q, \Psi}<\infty$, we have the following equivalent inequalities:

$$
\begin{aligned}
& \sum_{n=1}^{\infty} \int_{0}^{\infty} \frac{\operatorname{csch}\left(\rho\left(\frac{V_{n}}{U(x)}\right)^{\gamma}\right)}{e^{\alpha\left(\frac{V_{n}}{U(x)}\right)^{\gamma}}} a_{n} f(x) d x>k(\sigma)\|f\|_{p, \Phi_{-1}}\|a\|_{q, \Psi}, \\
& \sum_{n=1}^{\infty} \frac{v_{n}}{V_{n}^{1-p \sigma}}\left[\int_{0}^{\infty} \frac{\operatorname{csch}\left(\rho\left(\frac{V_{n}}{U(x)}\right)^{\gamma}\right)}{e^{\alpha\left(\frac{V_{n}}{U(x)}\right)^{\gamma}}} f(x) d x\right]^{p}>k(\sigma)\|f\|_{p, \Phi_{-1}}, \\
& \left\{\int_{0}^{\infty} \frac{\mu(x)}{U^{1+q \sigma}(x)}\left[\sum_{n=1}^{\infty} \frac{\operatorname{csch}\left(\rho\left(\frac{V_{n}}{U(x)}\right)^{\gamma}\right)}{e^{\alpha\left(\frac{V_{n}}{U(x)}\right)^{\gamma}}} a_{n}\right]^{q} d x\right\}^{\frac{1}{q}}>k(\sigma)\|a\|_{q, \Psi} ;
\end{aligned}
$$

(iii) for $0<p<1,0<\|f\|_{p, \Phi_{-1}},\|a\|_{q, \Psi}<\infty$, we have the following equivalent inequalities: 


$$
\begin{aligned}
& \sum_{n=1}^{\infty} \int_{0}^{\infty} \frac{\operatorname{csch}\left(\rho\left(\frac{V_{n}}{U(x)}\right)^{\gamma}\right)}{e^{\alpha\left(\frac{V_{n}}{U(x)}\right)^{\gamma}}} a_{n} f(x) d x>k(\sigma)\|f\|_{p, \widetilde{\Phi}_{-1}}\|a\|_{q, \Psi} \\
& \sum_{n=1}^{\infty} \frac{v_{n}}{V_{n}^{1-p \sigma}}\left[\int_{0}^{\infty} \frac{\operatorname{csch}\left(\rho\left(\frac{V_{n}}{U(x)}\right)^{\gamma}\right)}{e^{\alpha\left(\frac{V_{n}}{U(x)}\right)^{\gamma}}} f(x) d x\right]^{p}>k(\sigma)\|f\|_{p, \widetilde{\Phi}_{-1}}, \\
& \left\{\int_{0}^{\infty} \frac{\left(1-\theta_{-1}(\sigma, x)\right)^{1-q} \mu(x)}{U^{1+q \sigma}(x)}\left[\sum_{n=1}^{\infty} \frac{\operatorname{csch}\left(\rho\left(\frac{V_{n}}{U(x)}\right)^{\gamma}\right)}{e^{\alpha\left(\frac{V_{n}}{U(x)}\right)^{\gamma}}} a_{n}\right]^{q} d x\right\}^{\frac{1}{q}} \\
& >k(\sigma)\|a\|_{q, \Psi} \text {. }
\end{aligned}
$$

The above inequalities involve the best possible constant factor $k(\sigma)$. For $\alpha=\rho$ in Theorem 2-4, we have

Corollary 4. If $\rho>0,0<\gamma<\sigma \leq 1$,

$$
K(\sigma):=\frac{2 \Gamma\left(\frac{\sigma}{\gamma}\right) \zeta\left(\frac{\sigma}{\gamma}\right)}{\gamma(2 \rho)^{\sigma / \gamma}}
$$

there exists $n_{0} \in \mathbf{N}$, such that $v_{n} \geq v_{n+1}\left(n \in\left\{n_{0}, n_{0}+1, \cdots\right\}\right)$, and $U(\infty)=V(\infty)=$ $\infty$, then

(i) for $p>1,0<\|f\|_{p, \Phi_{\delta}},\|a\|_{q, \widetilde{\Psi}}<\infty$, we have the following equivalent inequalities with the best possible constant factor $K(\sigma)$ :

$$
\begin{gathered}
\sum_{n=1}^{\infty} \int_{0}^{\infty} \frac{\operatorname{csch}\left(\rho\left(U^{\delta}(x) \widetilde{V}_{n}\right)^{\gamma}\right)}{e^{\rho\left(U^{\delta}(x) \widetilde{V}_{n}\right)^{\gamma}}} a_{n} f(x) d x<K(\sigma)\|f\|_{p, \Phi_{\delta}}\|a\|_{q, \widetilde{\Psi}} \\
\sum_{n=1}^{\infty} \frac{v_{n}}{\widetilde{V}_{n}^{1-p \sigma}}\left[\int_{0}^{\infty} \frac{\operatorname{csch}\left(\rho\left(U^{\delta}(x) \widetilde{V}_{n}\right)^{\gamma}\right)}{e^{\rho\left(U^{\delta}(x) \widetilde{V}_{n}\right)^{\gamma}}} f(x) d x\right]^{p}<K(\sigma)\|f\|_{p, \Phi_{\delta}} \\
\left\{\int_{0}^{\infty} \frac{\mu(x)}{U^{1-q \delta \sigma}(x)}\left[\sum_{n=1}^{\infty} \frac{\operatorname{csch}\left(\rho\left(U^{\delta}(x) \widetilde{V}_{n}\right)^{\gamma}\right)}{e^{\rho\left(U^{\delta}(x) \widetilde{V}_{n}\right)^{\gamma}}} a_{n}\right]^{q} d x\right\}^{\frac{1}{q}}<K(\sigma)\|a\|_{q, \widetilde{\Psi}} .
\end{gathered}
$$

(ii) for $p<0,0<\|f\|_{p, \Phi_{\delta}},\|a\|_{q, \widetilde{\Psi}}<\infty$, we have the following equivalent inequalities with the best possible constant factor $K(\sigma)$ :

$$
\begin{aligned}
& \sum_{n=1}^{\infty} \int_{0}^{\infty} \frac{\operatorname{csch}\left(\rho\left(U^{\delta}(x) \widetilde{V}_{n}\right)^{\gamma}\right)}{e^{\rho\left(U^{\delta}(x) \widetilde{V}_{n}\right)^{\gamma}}} a_{n} f(x) d x>K(\sigma)\|f\|_{p, \Phi_{\delta}}\|a\|_{q, \widetilde{\Psi}}, \\
& \sum_{n=1}^{\infty} \frac{v_{n}}{\widetilde{V}_{n}^{1-p \sigma}}\left[\int_{0}^{\infty} \frac{\operatorname{csch}\left(\rho\left(U^{\delta}(x) \widetilde{V}_{n}\right)^{\gamma}\right)}{e^{\rho\left(U^{\delta}(x) \widetilde{V}_{n}\right)^{\gamma}}} f(x) d x\right]^{p}>K(\sigma)\|f\|_{p, \Phi_{\delta}},
\end{aligned}
$$




$$
\left\{\int_{0}^{\infty} \frac{\mu(x)}{U^{1-q \delta \sigma}(x)}\left[\sum_{n=1}^{\infty} \frac{\operatorname{csch}\left(\rho\left(U^{\delta}(x) \widetilde{V}_{n}\right)^{\gamma}\right)}{e^{\rho\left(U^{\delta}(x) \widetilde{V}_{n}\right)^{\gamma}}} a_{n}\right]^{q} d x\right\}^{\frac{1}{q}}>K(\sigma)\|a\|_{q, \widetilde{\Psi}}
$$

(iii) for $0<p<1,0<\|f\|_{p, \Phi_{\delta}},\|a\|_{q, \widetilde{\Psi}}<\infty$, we have the following equivalent inequalities with the best possible constant factor $K(\sigma)$ :

$$
\begin{aligned}
& \sum_{n=1}^{\infty} \int_{0}^{\infty} \frac{\operatorname{csch}\left(\rho\left(U^{\delta}(x) \widetilde{V}_{n}\right)^{\gamma}\right)}{e^{\rho\left(U^{\delta}(x) \widetilde{V}_{n}\right)^{\gamma}}} a_{n} f(x) d x>K(\sigma)\|f\|_{p, \widetilde{\Phi}_{\delta}}\|a\|_{q, \widetilde{\Psi}}, \\
& \sum_{n=1}^{\infty} \frac{v_{n}}{\widetilde{V}_{n}^{1-p \sigma}}\left[\int_{0}^{\infty} \frac{\operatorname{csch}\left(\rho\left(U^{\delta}(x) \widetilde{V}_{n}\right)^{\gamma}\right)}{e^{\rho\left(U^{\delta}(x) \widetilde{V}_{n}\right)^{\gamma}}} f(x) d x\right]^{p}>K(\sigma)\|f\|_{p, \widetilde{\Phi}_{\delta},} \\
& \quad\left\{\int_{0}^{\infty} \frac{\left(1-\theta_{\delta}(\sigma, x)\right)^{1-q} \mu(x)}{U^{1-q \delta \sigma}(x)}\left[\sum_{n=1}^{\infty} \frac{\operatorname{csch}\left(\rho\left(U^{\delta}(x) \widetilde{V}_{n}\right)^{\gamma}\right)}{e^{\rho\left(U^{\delta}(x) \widetilde{V}_{n}\right)^{\gamma}}} a_{n}\right]^{q} d x\right\}^{\frac{1}{q}} \\
& >K(\sigma)\|a\|_{q, \widetilde{\Psi}} \cdot
\end{aligned}
$$

In particular, for $\gamma=\frac{\sigma}{2}, \theta_{\delta}(\sigma, x)=O\left((U(x))^{\frac{\delta \sigma}{2}}\right)$,

(i) for $p>1$, we have the following equivalent inequalities with the best possible constant factor $\frac{\pi^{2}}{6 \sigma \rho^{2}}$ :

$$
\begin{gathered}
\sum_{n=1}^{\infty} \int_{0}^{\infty} \frac{\operatorname{csch}\left(\rho\left(U^{\delta}(x) \widetilde{V}_{n}\right)^{\sigma / 2}\right)}{e^{\rho\left(U^{\delta}(x) \widetilde{V}_{n}\right)^{\sigma / 2}}} a_{n} f(x) d x<\frac{\pi^{2}}{6 \sigma \rho^{2}}\|f\|_{p, \Phi_{\delta}}\|a\|_{q, \widetilde{\Psi}}, \\
\sum_{n=1}^{\infty} \frac{v_{n}}{\widetilde{V}_{n}^{1-p \sigma}}\left[\int_{0}^{\infty} \frac{\operatorname{csch}\left(\rho\left(U^{\delta}(x) \widetilde{V}_{n}\right)^{\sigma / 2}\right)}{e^{\rho\left(U^{\delta}(x) \widetilde{V}_{n}\right)^{\sigma / 2}}} f(x) d x\right]^{p}<\frac{\pi^{2}}{6 \sigma \rho^{2}}\|f\|_{p, \Phi_{\delta}} \\
\left\{\int_{0}^{\infty} \frac{\mu(x)}{U^{1-q \delta \sigma}(x)}\left[\sum_{n=1}^{\infty} \frac{\operatorname{csch}\left(\rho\left(U^{\delta}(x) \widetilde{V}_{n}\right)^{\sigma / 2}\right)}{e^{\rho\left(U^{\delta}(x) \widetilde{V}_{n}\right)^{\sigma / 2}}} a_{n}\right]^{q} d x\right\}^{\frac{1}{q}}<\frac{\pi^{2}}{6 \sigma \rho^{2}}\|a\|_{q, \widetilde{\Psi}}
\end{gathered}
$$

(ii) for $p<0$,we have the following equivalent inequalities with the best possible constant factor $\frac{\pi^{2}}{6 \sigma \rho^{2}}$ :

$$
\begin{gathered}
\sum_{n=1}^{\infty} \int_{0}^{\infty} \frac{\operatorname{csch}\left(\rho\left(U^{\delta}(x) \widetilde{V}_{n}\right)^{\sigma / 2}\right)}{e^{\rho\left(U^{\delta}(x) \widetilde{V}_{n}\right)^{\sigma / 2}}} a_{n} f(x) d x>\frac{\pi^{2}}{6 \sigma \rho^{2}}\|f\|_{p, \Phi_{\delta}}\|a\|_{q, \widetilde{\Psi}} \\
\sum_{n=1}^{\infty} \frac{v_{n}}{\widetilde{V}_{n}^{1-p \sigma}}\left[\int_{0}^{\infty} \frac{\operatorname{csch}\left(\rho\left(U^{\delta}(x) \widetilde{V}_{n}\right)^{\sigma / 2}\right)}{e^{\rho\left(U^{\delta}(x) \widetilde{V}_{n}\right)^{\sigma / 2}}} f(x) d x\right]^{p}>\frac{\pi^{2}}{6 \sigma \rho^{2}}\|f\|_{p, \Phi_{\delta}} \\
\left\{\int_{0}^{\infty} \frac{\mu(x)}{U^{1-q \delta \sigma}(x)}\left[\sum_{n=1}^{\infty} \frac{\operatorname{csch}\left(\rho\left(U^{\delta}(x) \widetilde{V}_{n}\right)^{\sigma / 2}\right)}{e^{\rho\left(U^{\delta}(x) \widetilde{V}_{n}\right)^{\sigma / 2}}} a_{n}\right]^{q} d x\right\}^{\frac{1}{q}}>\frac{\pi^{2}}{6 \sigma \rho^{2}}\|a\|_{q, \widetilde{\Psi}}
\end{gathered}
$$


(iii) for $0<p<1$, we have the following equivalent inequalities with the best possible constant factor $\frac{\pi^{2}}{6 \sigma \rho^{2}}$ :

$$
\begin{aligned}
& \sum_{n=1}^{\infty} \int_{0}^{\infty} \frac{\operatorname{csch}\left(\rho\left(U^{\delta}(x) \widetilde{V}_{n}\right)^{\sigma / 2}\right)}{e^{\rho\left(U^{\delta}(x) \widetilde{V}_{n}\right)^{\sigma / 2}}} a_{n} f(x) d x>\frac{\pi^{2}}{6 \sigma \rho^{2}}\|f\|_{p, \widetilde{\Phi}_{\delta}}\|a\|_{q, \widetilde{\Psi}}, \\
& \sum_{n=1}^{\infty} \frac{v_{n}}{\widetilde{V}_{n}^{1-p \sigma}}\left[\int_{0}^{\infty} \frac{\operatorname{csch}\left(\rho\left(U^{\delta}(x) \widetilde{V}_{n}\right)^{\sigma / 2}\right)}{e^{\rho\left(U^{\delta}(x) \widetilde{V}_{n}\right)^{\sigma / 2}}} f(x) d x\right]^{p}>\frac{\pi^{2}}{6 \sigma \rho^{2}}\|f\|_{p, \widetilde{\Phi}_{\delta}}, \\
& \quad\left\{\int_{0}^{\infty} \frac{\left(1-\theta_{\delta}(\sigma, x)\right)^{1-q} \mu(x)}{U^{1-q \delta \sigma}(x)}\left[\sum_{n=1}^{\infty} \frac{\operatorname{csch}\left(\rho\left(U^{\delta}(x) \widetilde{V}_{n}\right)^{\sigma / 2}\right)}{e^{\rho\left(U^{\delta}(x) \widetilde{V}_{n}\right)^{\sigma / 2}}} a_{n}\right]^{q} d x\right\}^{\frac{1}{q}} \\
& >\frac{\pi^{2}}{6 \sigma \rho^{2}}\|a\|_{q, \widetilde{\Psi}} .
\end{aligned}
$$

For $\alpha=0, \gamma=\frac{\sigma}{2}, \theta_{\delta}(\sigma, x)=O\left((U(x))^{\frac{\delta \sigma}{2}}\right)$ in Theorem 2-4, we have

Corollary 5. If $\rho>0,0<\sigma \leq 1$, there exists $n_{0} \in \mathbf{N}$, such that $v_{n} \geq v_{n+1}(n \in$ $\left.\left\{n_{0}, n_{0}+1, \cdots\right\}\right)$, and $U(\infty)=V(\infty)=\infty$, then

(i) for $p>1,0<\|f\|_{p, \Phi_{\delta}},\|a\|_{q, \widetilde{\Psi}}<\infty$, we have the following equivalent inequalities with the best possible constant factor $\frac{\pi^{2}}{2 \sigma \rho^{2}}$ :

$$
\begin{aligned}
& \sum_{n=1}^{\infty} \int_{0}^{\infty} \operatorname{csch}\left(\rho\left(U^{\delta}(x) \widetilde{V}_{n}\right)^{\frac{\sigma}{2}}\right) a_{n} f(x) d x<\frac{\pi^{2}}{2 \sigma \rho^{2}}\|f\|\left\|_{p, \Phi_{\delta}}\right\| a \|_{q, \widetilde{\Psi}}, \\
& \sum_{n=1}^{\infty} \frac{v_{n}}{\widetilde{V}_{n}^{1-p \sigma}}\left[\int_{0}^{\infty} \operatorname{csch}\left(\rho\left(U^{\delta}(x) \widetilde{V}_{n}\right)^{\frac{\sigma}{2}}\right) f(x) d x\right]^{p}<\frac{\pi^{2}}{2 \sigma \rho^{2}}\|f\|_{p, \Phi_{\delta}}, \\
& \left\{\int_{0}^{\infty} \frac{\mu(x)}{U^{1-q \delta \sigma}(x)}\left[\sum_{n=1}^{\infty} \operatorname{csch}\left(\rho\left(U^{\delta}(x) \widetilde{V}_{n}\right)^{\frac{\sigma}{2}}\right) a_{n}\right]^{q} d x\right\}^{\frac{1}{q}}<\frac{\pi^{2}}{2 \sigma \rho^{2}}\|a\|_{q, \widetilde{\Psi}} ;
\end{aligned}
$$

(ii) for $p<0,0<\|f\|_{p, \Phi_{\delta}},\|a\|_{q, \widetilde{\Psi}}<\infty$, we have the following equivalent inequalities with the best possible constant factor $\frac{\pi^{2}}{2 \sigma \rho^{2}}$ :

$$
\begin{aligned}
& \sum_{n=1}^{\infty} \int_{0}^{\infty} \operatorname{csch}\left(\rho\left(U^{\delta}(x) \widetilde{V}_{n}\right)^{\frac{\sigma}{2}}\right) a_{n} f(x) d x>\frac{\pi^{2}}{2 \sigma \rho^{2}}\|f\|_{p, \Phi_{\delta}}\|a\|_{q, \widetilde{\Psi}}, \\
& \sum_{n=1}^{\infty} \frac{v_{n}}{\widetilde{V}_{n}^{1-p \sigma}}\left[\int_{0}^{\infty} \operatorname{csch}\left(\rho\left(U^{\delta}(x) \widetilde{V}_{n}\right)^{\frac{\sigma}{2}}\right) f(x) d x\right]^{p}>\frac{\pi^{2}}{2 \sigma \rho^{2}}\|f\|_{p, \Phi_{\delta}}, \\
& \left\{\int_{0}^{\infty} \frac{\mu(x)}{U^{1-q \delta \sigma}(x)}\left[\sum_{n=1}^{\infty} \operatorname{csch}\left(\rho\left(U^{\delta}(x) \widetilde{V}_{n}\right)^{\frac{\sigma}{2}}\right) a_{n}\right]^{q} d x\right\}^{\frac{1}{q}}>\frac{\pi^{2}}{2 \sigma \rho^{2}}\|a\|_{q, \widetilde{\Psi}} ;
\end{aligned}
$$


(iii) for $0<p<1,0<\|f\|_{p, \Phi_{\delta}},\|a\|_{q, \widetilde{\Psi}}<\infty$, we have the following equivalent inequalities with the best possible constant factor $\frac{\pi^{2}}{2 \sigma \rho^{2}}$ :

$$
\begin{aligned}
& \quad \sum_{n=1}^{\infty} \int_{0}^{\infty} \operatorname{csch}\left(\rho\left(U^{\delta}(x) \widetilde{V}_{n}\right)^{\frac{\sigma}{2}}\right) a_{n} f(x) d x>\frac{\pi^{2}}{2 \sigma \rho^{2}}\|f\|_{p, \widetilde{\Phi}_{\delta}}\|a\|_{q, \widetilde{\Psi}}, \\
& \sum_{n=1}^{\infty} \frac{v_{n}}{\widetilde{V}_{n}^{1-p \sigma}}\left[\int_{0}^{\infty} \operatorname{csch}\left(\rho\left(U^{\delta}(x) \widetilde{V}_{n}\right)^{\frac{\sigma}{2}}\right) f(x) d x\right]^{p}>\frac{\pi^{2}}{2 \sigma \rho^{2}}\|f\|_{p, \widetilde{\Phi}_{\delta},} \\
& \quad\left\{\int_{0}^{\infty} \frac{\left(1-\theta_{\delta}(\sigma, x)\right)^{1-q} \mu(x)}{U^{1-q \delta \sigma}(x)}\left[\sum_{n=1}^{\infty} \operatorname{csch}\left(\rho\left(U^{\delta}(x) \widetilde{V}_{n}\right)^{\frac{\sigma}{2}}\right) a_{n}\right]^{q} d x\right\}^{\frac{1}{q}} \\
& >\frac{\pi^{2}}{2 \sigma \rho^{2}}\|a\|_{q, \widetilde{\Psi}} .
\end{aligned}
$$

Remark 2. (i) For $\mu(x)=v_{n}=1$ in (54), we have the following inequality with the best possible constant factor $k(\sigma)$ :

$$
\begin{aligned}
& \sum_{n=1}^{\infty} \int_{0}^{\infty} \frac{\operatorname{csch}\left(\rho\left(x^{\delta} n\right)^{\gamma}\right)}{e^{\alpha\left(x^{\delta} n\right)^{\gamma}}} a_{n} f(x) d x \\
< & k(\sigma)\left[\int_{0}^{\infty} x^{p(1-\delta \sigma)-1} f^{p}(x) d x\right]^{\frac{1}{p}}\left[\sum_{n=1}^{\infty} n^{q(1-\sigma)-1} a_{n}^{q}\right]^{\frac{1}{q}} .
\end{aligned}
$$

In particular, for $\delta=1$, we have the following inequality with the non-homogeneous kernel:

$$
\begin{aligned}
& \sum_{n=1}^{\infty} \int_{0}^{\infty} \frac{\operatorname{csch}\left(\rho(x n)^{\gamma}\right)}{e^{\alpha(x n)^{\gamma}}} a_{n} f(x) d x \\
< & k(\sigma)\left[\int_{0}^{\infty} x^{p(1-\sigma)-1} f^{p}(x) d x\right]^{\frac{1}{p}}\left[\sum_{n=1}^{\infty} n^{q(1-\sigma)-1} a_{n}^{q}\right]^{\frac{1}{q}} ;
\end{aligned}
$$

for $\delta=-1$, we have the following inequality with the homogeneous kernel:

$$
\begin{aligned}
& \sum_{n=1}^{\infty} \int_{0}^{\infty} \frac{\operatorname{csch}\left(\rho\left(\frac{n}{x}\right)^{\gamma}\right)}{e^{\alpha\left(\frac{n}{x}\right)^{\gamma}}} a_{n} f(x) d x \\
< & k(\sigma)\left[\int_{0}^{\infty} x^{p(1+\sigma)-1} f^{p}(x) d x\right]^{\frac{1}{p}}\left[\sum_{n=1}^{\infty} n^{q(1-\sigma)-1} a_{n}^{q}\right]^{\frac{1}{q}} .
\end{aligned}
$$

(ii) For $\mu(x)=v_{n}=1, \widetilde{v}_{n}=\tau \in\left(0, \frac{1}{2}\right]$ in $[22)$, we have the following more accurate inequality than 81 with the best possible constant factor $k(\sigma)$ : 


$$
\begin{aligned}
& \sum_{n=1}^{\infty} \int_{0}^{\infty} \frac{\operatorname{csch}\left(\rho\left[x^{\delta}(n-\tau)\right]^{\gamma}\right)}{e^{\left.\alpha\left(x^{\delta}(n-\tau)\right]^{\gamma}\right)^{\gamma}}} a_{n} f(x) d x \\
< & k(\sigma)\left[\int_{0}^{\infty} x^{p(1-\delta \sigma)-1} f^{p}(x) d x\right]^{\frac{1}{p}}\left[\sum_{n=1}^{\infty}(n-\tau)^{q(1-\sigma)-1} a_{n}^{q}\right]^{\frac{1}{q}} .
\end{aligned}
$$

In particular, for $\delta=1$, we have the following inequality with the non-homogeneous kernel:

$$
\begin{aligned}
& \sum_{n=1}^{\infty} \int_{0}^{\infty} \frac{\operatorname{csch}\left([x(n-\tau)]^{\gamma}\right)}{e^{\alpha\{[x(n-\tau)]\}^{\gamma}}} a_{n} f(x) d x \\
< & k(\sigma)\left[\int_{0}^{\infty} x^{p(1-\sigma)-1} f^{p}(x) d x\right]^{\frac{1}{p}}\left[\sum_{n=1}^{\infty}(n-\tau)^{q(1-\sigma)-1} a_{n}^{q}\right]^{\frac{1}{q}} ;
\end{aligned}
$$

for $\delta=-1$, we have the following inequality with the homogeneous kernel:

$$
\begin{aligned}
& \sum_{n=1}^{\infty} \int_{0}^{\infty} \frac{\operatorname{csch}\left(\rho\left(\frac{n-\tau}{x}\right)^{\gamma}\right)}{e^{\alpha\left(\frac{n-\tau}{x}\right)^{\gamma}}} a_{n} f(x) d x \\
< & k(\sigma)\left[\int_{0}^{\infty} x^{p(1+\sigma)-1} f^{p}(x) d x\right]^{\frac{1}{p}}\left[\sum_{n=1}^{\infty}(n-\tau)^{q(1-\sigma)-1} a_{n}^{q}\right]^{\frac{1}{q}} .
\end{aligned}
$$

We can still obtain a large number of other inequalities by using some special parameters in the above Theorems and Corollaries.

Acknowledgements. B. Yang: This work is supported by the National Natural Science Foundation of China (No. 61370186), and 2013 Knowledge Construction Special Foundation Item of Guangdong Institution of Higher Learning College and University (No. 2013KJCX0140).

\section{References}

1. Hardy G. H., Littlewood J. E., Pólya G.: Inequalities, Cambridge University Press, Cambridge (1934).

2. Mitrinović D. S., Pečarić J. E., Fink A. M.: Inequalities involving functions and their integrals and derivatives. Kluwer Academic Publishers, Boston (1991).

3. Yang B. C.: Hilbert-type integral inequalities. Bentham Science Publishers Ltd., The United Arab Emirates (2009).

4. Yang B. C.: Discrete Hilbert-type inequalities. Bentham Science Publishers Ltd., The United Arab Emirates (2011).

5. Yang B. C.: The norm of operator and Hilbert-type inequalities, Science Press, Beijin, China (2009). 
6. Yang B. C.: On Hilbert's integral inequality. Journal of Mathematical Analysis and Applications, 220, 778-785(1998).

7. Yang B. C.: An extension of a Hardy-Hilbert-type inequality. Journal of Guangdong University of Education, 35(3): 1-8 (2015).

8. Yang B. C., Brnetić I, Krnić M., Pečarić J. E.: Generalization of Hilbert and Hardy-Hilbert integral inequalities. Math. Ineq. and Appl., 8(2), 259-272 (2005).

9. Krnić M., Pečarić J. E.: Hilbert's inequalities and their reverses, Publ. Math. Debrecen, 67(34), 315-331 (2005).

10. Yang B. C., Rassias Th. M., On the way of weight coefficient and research for Hilbert-type inequalities, Math. Ineq. Appl., 6(4), 625-658 (2003).

11. Yang B. C., Rassias Th. M., On a Hilbert-type integral inequality in the subinterval and its operator expression. Banach J. Math. Anal., 4(2), 100-110 (2010).

12. Azar L.: On some extensions of Hardy-Hilbert's inequality and Applications. Journal of Inequalities and Applications, 2009, no. 546829.

13. Arpad B., Choonghong O.: Best constant for certain multilinear integral operator. Journal of Inequalities and Applications, 2006, no. 28582.

14. Kuang J. C., Debnath L.: On Hilbert's type inequalities on the weighted Orlicz spaces. Pacific J. Appl. Math., 1(1), 95-103 (2007).

15. Zhong W. Y.: The Hilbert-type integral inequality with a homogeneous kernel of Lambdadegree. Journal of Inequalities and Applications, 2008, no. 917392.

16. Hong Y.: On Hardy-Hilbert integral inequalities with some parameters. J. Ineq. in Pure \& Applied Math., 6(4), Art. 92, 1-10 (2005).

17. Zhong W. Y., Yang B. C.: On multiple Hardy-Hilbert's integral inequality with kernel. Journal of Inequalities and Applications, Vol. 2007, Art.ID 27962, 17 pages, doi: 10.1155/ 2007/27.

18. Yang B. C., Krnić M.: On the Norm of a Mult-dimensional Hilbert-type Operator, Sarajevo Journal of Mathematics, 7(20), 223-243(2011).

19. Krnić M., Pečarić J. E., Vuković P.: On some higher-dimensional Hilbert's and Hardy-Hilbert's type integral inequalities with parameters, Math. Inequal. Appl., 11,701-716 (2008).

20. Krnić M., Vuković P.: On a multidimensional version of the Hilbert-type inequality, Analysis Mathematica, 38, 291-303 (2012).

21. Rassias M. Th., Yang B. C.: On half-discrete Hilbert's inequality. Applied Mathematics and Computation, 220: 75-93 (2013).

22. Rassias M. Th., Yang B. C.: A multidimensional half-discrete Hilbert-type inequality and the Riemann zeta function, Applied Mathematics and Computation, 225: 263-277 (2013).

23. Rassias M. Th., Yang B. C.: On a multidimensional half - discrete Hilbert - type inequality related to the hyperbolic cotangent function, Applied Mathematics and Computation, 242: 800 -813 (2014).

24. Rassias M. Th., Yang B. C.: On a multidimensional Hilbert-type integral inequality associated to the gamma function, Applied Mathematics and Computation, 249: 408 - 418 (2014).

25. Yang B. C.: On a more accurate multidimensional Hilbert-type inequality with parameters. Mathematical Inequalities and Applications, 18(2): 429-441 (2015).

26. Yang B. C.: A mixed Hilbert-type inequality with a best constant factor. International Journal of Pure and Applied Mathematcs, 20(3), 319-328 (2005).

27. Yang B. C.: A half-discrete Hilbert-type inequality. Journal of Guangdong University of Education, 31(3), 1-7 (2011).

28. Zhong W. Y.: A mixed Hilbert-type inequality and its equivalent forms. Journal of Guangdong University of Education, 31(5), 18-22 (2011).

29. Zhong W. Y.: A half discrete Hilbert-type inequality and its equivalent forms. Journal of Guangdong University of Education, 32(5), 8-12 (2012).

30. Zhong J. H., Yang B. C.: On an extension of a more accurate Hilbert-type inequality. Journal of Zhejiang University (Science Edition), 35(2), 121-124 (2008).

31. Zhong J. H.: Two classes of half-discrete reverse Hilbert-type inequalities with a nonhomogeneous kernel. Journal of Guangdong University of Education, 32(5), 11-20 (2012). 
32. Zhong W. Y., Yang B. C.: A best extension of Hilbert inequality involving several parameters. Journal of Jinan University (Natural Science), 28(1), 20-23 (2007).

33. Zhong W. Y., Yang B. C.: A reverse Hilbert's type integral inequality with some parameters and the equivalent forms. Pure and Applied Mathematics, 24(2), 401-407 (2008).

34. Zhong W. Y., Yang B. C.: On multiple Hardy-Hilbert's integral inequality with kernel. Journal of Inequalities and Applications, Vol. 2007, Art.ID 27962, 17 pages.

35. Yang B. C., Chen Q.: A half-discrete Hilbert-type inequality with a homogeneous kernel and an extension. Journal of Inequalities and Applications, 124 (2011).

36. Yang B. C.: A half-discrete Hilbert-type inequality with a non-homogeneous kernel and two variables. Mediterranean Journal of Methematics, 10, 677-692 (2013).

37. Yang B. C. : Two types of multiple half-discrete Hilbert-type inequalities. Lambert Academic Publishing, Germany (2012).

38. Yang B. C. : Topics on half-discrete Hilbert-type inequalities. Lambert Academic Publishing, Germany (2013).

39. Yang B. C., Debnath L.: Half-discrete Hilbert-type inequalities. World Scientific Publishing, Singapore (2014)

40. Zhong Y. Q.: Introduction to Complex functions. Higher Education Press, Beijing, China (2003).

41. Wang Z. Q, Guo D. R.: Introduction to special functions, Science Press, Beijing, China (1979).

42. Kuang J. C.: Real analysis and Functional analysis. Higher Education Press, Beijing, China (2014)

43. Kuang J. C.: Applied inequalities. Shangdong Science Technic Press, Jinan, China (2004). 